\title{
Review
}

\section{Reaching and Grasping Movements in Parkinson's Disease: A Review}

\author{
Alessio Fasano $^{\mathrm{a}, \mathrm{b}, *}$, Alberto Mazzoni ${ }^{\mathrm{a}, \mathrm{b}, 1}$ and Egidio Falotico ${ }^{\mathrm{a}, \mathrm{b}, 1, *}$ \\ ${ }^{a}$ The BioRobotics Institute, Scuola Superiore Sant'Anna, Pisa, Italy \\ ${ }^{\mathrm{b}}$ Department of Excellence in Robotics and AI, Scuola Superiore Sant'Anna, Pisa, Italy
}

Accepted 15 February 2022

Pre-press 2 March 2022

\begin{abstract}
Parkinson's disease (PD) is known to affect the brain motor circuits involving the basal ganglia (BG) and to induce, among other signs, general slowness and paucity of movements. In upper limb movements, PD patients show a systematic prolongation of movement duration while maintaining a sufficient level of endpoint accuracy. PD appears to cause impairments not only in movement execution, but also in movement initiation and planning, as revealed by abnormal preparatory activity of motor-related brain areas. Grasping movement is affected as well, particularly in the coordination of the hand aperture with the transport phase. In the last fifty years, numerous behavioral studies attempted to clarify the mechanisms underlying these anomalies, speculating on the plausible role that the BG-thalamo-cortical circuitry may play in normal and pathological motor control. Still, many questions remain open, especially concerning the management of the speed-accuracy tradeoff and the online feedback control. In this review, we summarize the literature results on reaching and grasping in parkinsonian patients. We analyze the relevant hypotheses on the origins of dysfunction, by focusing on the motor control aspects involved in the different movement phases and the corresponding role played by the BG. We conclude with an insight into the innovative stimulation techniques and computational models recently proposed, which might be helpful in further clarifying the mechanisms through which PD affects reaching and grasping movements.
\end{abstract}

Keywords: Reach and grasp, Parkinson's disease, motor control, upper limb aiming movements, bradykinesia, hypokinesia, akinesia, basal ganglia, computational modelling, brain stimulation

\section{OVERVIEW}

Parkinson's disease (PD) has a very broad spectrum of clinical manifestations, among which a diminished range of motion appears to be quite common. More specifically, PD patients clinically present reduced amplitude, or hypokinesia, reduced speed, or bradykinesia, and impaired initiation (i.e., increased latency of onset), or akinesia, of voluntary movement. These signs are usually accompanied

\footnotetext{
${ }^{1}$ These authors share an equal senior author contribution.

*Correspondence to: Alessio Fasano and Egidio Falotico, The BioRobotics Institute, Scuola Superiore Sant'Anna, Polo Sant'Anna Valdera, Viale Rinaldo Piaggio, 34, 56025 Pontedera (PI), Italy. Tel.: +39 050883 457; E-mails: a.fasano@ santannapisa.it and e.falotico@santannapisa.it
}

by stiffness of muscle tone (e.g., increased resistance to passive movement of a limb) and tremor at rest. Anomalies of activity patterns in electromyography (EMG) [1] and electroencephalography (EEG) [2], and an impaired proprioception [3] during upper limb movements have been early reported. While most of the current efforts have been put on unraveling the mechanisms of the freezing of gait [4], or other posture and balance problems [5], many questions concerning the origins of malfunctions in controlling arm and hand movements have remained unanswered.

Individuals with PD must face several challenges in complex activities of daily living involving the upper limb, such as reaching and grasping. Reaching out for an object requires effective spatio-temporal coordination of the joints of the upper limb through 
appropriate and efficient muscular activation, predictive synchronization of the hand transport with the fingers' aperture closure (i.e., when hand aperture begins to close), but also online processing of sensory information, both proprioceptive and visual. Generally, motor faults of PD patients' upper limb are clinically examined, diagnosed and tracked with simplified tasks, such as finger tapping and alternating hand motions, e.g., supination/pronation [6]. However, several behavioral studies have reported much more than a simple decrease of motion rate in repetitive movements and complex issues on the upper limb motor control in PD have come out [7-9]. In this regard, the kinematic analysis of the reach-to-grasp movement has been used to characterize different forms of parkinsonism [10].
There are several hypotheses about the dynamics of motor dysfunction in PD. This disease predominately affects the area of dopaminergic neurons of the substantia nigra pars compacta in the midbrain, leading to imbalances in the cortico-basal ganglia (BG) circuitries related to movement control, illustrated in Fig. 1. Hyperactivity of the indirect pathway compared to the direct pathway (see Fig. 1 for definitions), which leads to decreased excitation of the motor cortex, has been proposed as major responsible for the mentioned motor signs $[11,12]$. In the classical view, impaired BG cause inability to activate a selected, and appropriate, motor program or failure to inhibit competing motor programs [13]. A poor signal-to-noise ratio of the neural signal processing occurring in the BG has been an alternative view [14],

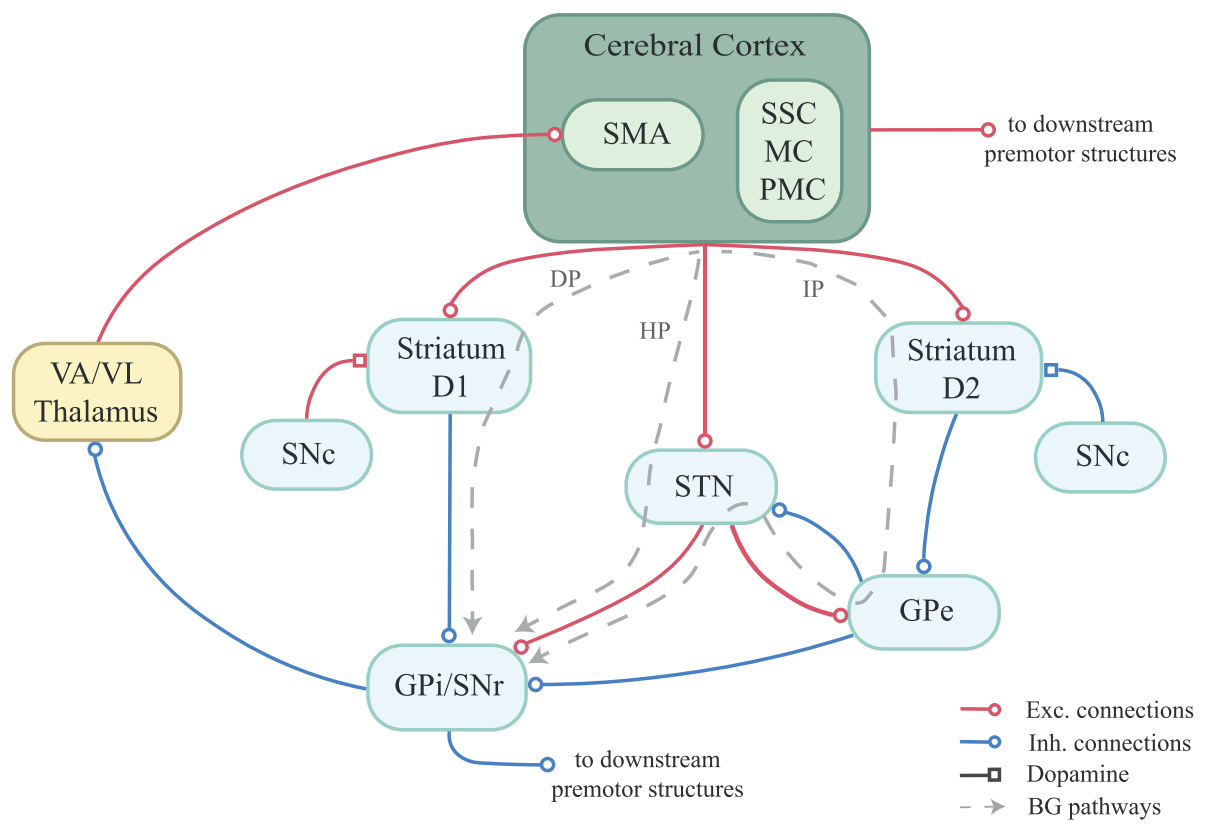

Fig. 1. Cortico-basal ganglia motor circuit. The depicted cortico-thalamic-basal ganglia (BG) motor circuit is inspired by the model of DeLong and colleagues $[11,12]$. BG consist of several interconnected subcortical nuclei. Striatum is the input structure. It receives input from almost the entire cerebral cortex. In particular, somatosensory (SSC), motor (MC) and premotor (PMC) cortices and supplementary motor area (SMA) are of interest for motor functions. Two sets of cells in the striatum can be distinguished on the basis of the type of dopamine receptors - D1 and D2 - expressed. They give rise to the direct and indirect pathways, respectively. The direct pathway (DP) brings straightforwardly to the output structures of the BG, globus pallidus internal segment (GPi) or the substantia nigra pars reticulata (SNr). The indirect pathway (IP) involves the globus pallidus external segment (GPe) and the subthalamic nucleus (STN), before getting to the GPi/SNr. An hyperdirect pathway (HP) sees a direct cortical excitation of the STN, bypassing the striatum. The STN sends excitatory projections to GPi/SNr. The output from GPi/SNr is inhibitory and projects to the ventral anterior and ventrolateral (VA/VL) thalamus, which excites the motor areas in the cortex, closing the loop. This output most influences SMA function. Gpi and SNr project also to the brainstem premotor structures, in particular the pedunculopontine nucleus, and to the superior colliculus. Overall, increased activity in the DP facilitates movements, releasing the thalamus from tonic inhibition of the GPi/SNr, while increased activity in the IP inhibits movements, by disinhibiting the GPi/SNr. The HP seems to contribute to early stopping of competing motor programs [247]. The balance between the pathways is affected by the dopaminergic projection from the substantia nigra pars compacta $(\mathrm{SNc})$ to the striatum: the release of dopamine in the DP increases the responsiveness of the D1 striatal cells to corticostriatal input, while the release of dopamine in the IP decreases the responsiveness of the D2 striatal cells. In hypokinetic movement disorder, such as Parkinson's disease, the degeneration of the nigrostriatal dopamine projections let the IP overpower the DP, which results in stronger inhibition of the thalamus. 
corroborated by the observed excessive synchronization in the neuronal firing of the subthalamic nucleus (STN)-external globus pallidus (GPe) network [15].

In the following, we will review the literature concerning reaching and grasping in the PD population, in the attempt to summarize all the major neuroscientific results and clarify the potential mechanisms of dysfunction underlying the reported anomalies. We will start from the behavioral studies on execution of upper limb discrete aiming movements with the observations of the PD signs, the issues around the speed-accuracy tradeoff, the hypotheses on bradykinesia and hypokinesia, the paradoxical movements and the impact of sensory cues on PD patients' performances. Aspects of both feed-forward planning and online feedback control will be taken into account. Next, we will examine the motor initiation and preparation, and the underlying anomalies in task-related brain activity. We will then discuss the PD patients' impairments in reach-to-grasp movement coordination. The last section will concern the recent studies on stimulation and computational modelling, which brought about novel working methods to formulate or test hypotheses on the neural substrates and the genesis of parkinsonian abnormal motor behaviors. Conclusive remarks will suggest potential directions to take for improving our understanding of the motor control aspects of this disease.

\section{ANOMALIES IN EXECUTION OF UPPER LIMB TASKS}

In a planar reaching task, or in aiming tasks with a cursor, the goal is to acquire a target at a predefined spatial distance through flexion/extension of the arm and/or forearm (Fig. 2). In these tasks normal subjects produce a rapid movement in the target direction with an accuracy of about $10 \%$ and lasting less than the average reaction time (less than half of a second for a few decimeters) $[8,16]$. When the target is sufficiently far and the task duration sufficiently long, this movement may be followed by a secondary corrective movement to adjust the trajectory towards the goal. Correction entails processing feedback (e.g., visual) information to voluntary control the execution, thus spending more than a reaction time to complete the movement [16]. Aiming movements can be therefore seen as two-component motor schemes in which an initial plan is executed ballistically and is followed by a closed-loop adjustment if the time is enough [16]. When varying the amplitude to cover, healthy subjects do not only show a consistent endpoint accuracy, but they also vary the velocity of execution to keep the duration fairly constant [17]. This reveals a selection of a specific, preprogrammed set of motor parameters for that particular aim.

In 1975 and 1976, Flowers reported a distortion of this mechanism in patients with PD performing pointing with a joystick [7, 8]. First, these subjects took longer to initiate the task. Then, as reported also previously in [18], they did not produce efficient ballistic movements but rather tended to move at constant, low speed whatever the target distance. The rate of movement was steady across conditions, not its duration, thus farthest targets were reached in significantly longer periods. The endpoint accuracy was excessively affected by increasing velocity and, when the starting point or the response marker were removed from the screen, patients made shorter and smaller movements. A general inconsistency of execution was revealed by a much larger than normal variance of speed and error. Furthermore, their performance did not considerably improve with practice. To explain his results, Flowers' proposal was that PD patients lose the ability to predetermine the correct size of the movement that would fit the required amplitude. In this view, PD disrupts the possibility of successful open-loop approaches, forcing patients to rely on a closed-loop form of control, i.e., on corrective mechanisms to fulfill the accuracy constraint. For distant targets, the consecutive online adjustments cause an increased duration of the task and the manifestation of bradykinesia.

The EMG recordings of Hallet et al. [1, 19] clarified the mechanisms underlying the kinematic observations of Flowers. For ballistic hand movements, healthy subjects employ a triphasic (agonistantagonist-agonist contractions) pattern of muscle activity characterized by a constant duration of each single phase, independently from the target distance, so that the magnitude of the bursts must be increased to reach a farther target [20]. In single-joint movements, patients can produce the normal triphasic pattern, but the amount of EMG activity for each burst in the cycle is insufficient, so that a longer distance requires additional cycles [19]. In the works of Hallett and colleagues, the size of the first agonist burst appeared to be particularly reduced in the PD group, suggesting a possible saturation of the process generating the EMG burst. Thus, the fundamental problem seemed to be the appropriate energization of the specific set of muscles for that particular movement [19]. If the first agonist activity is limited, the patient's 


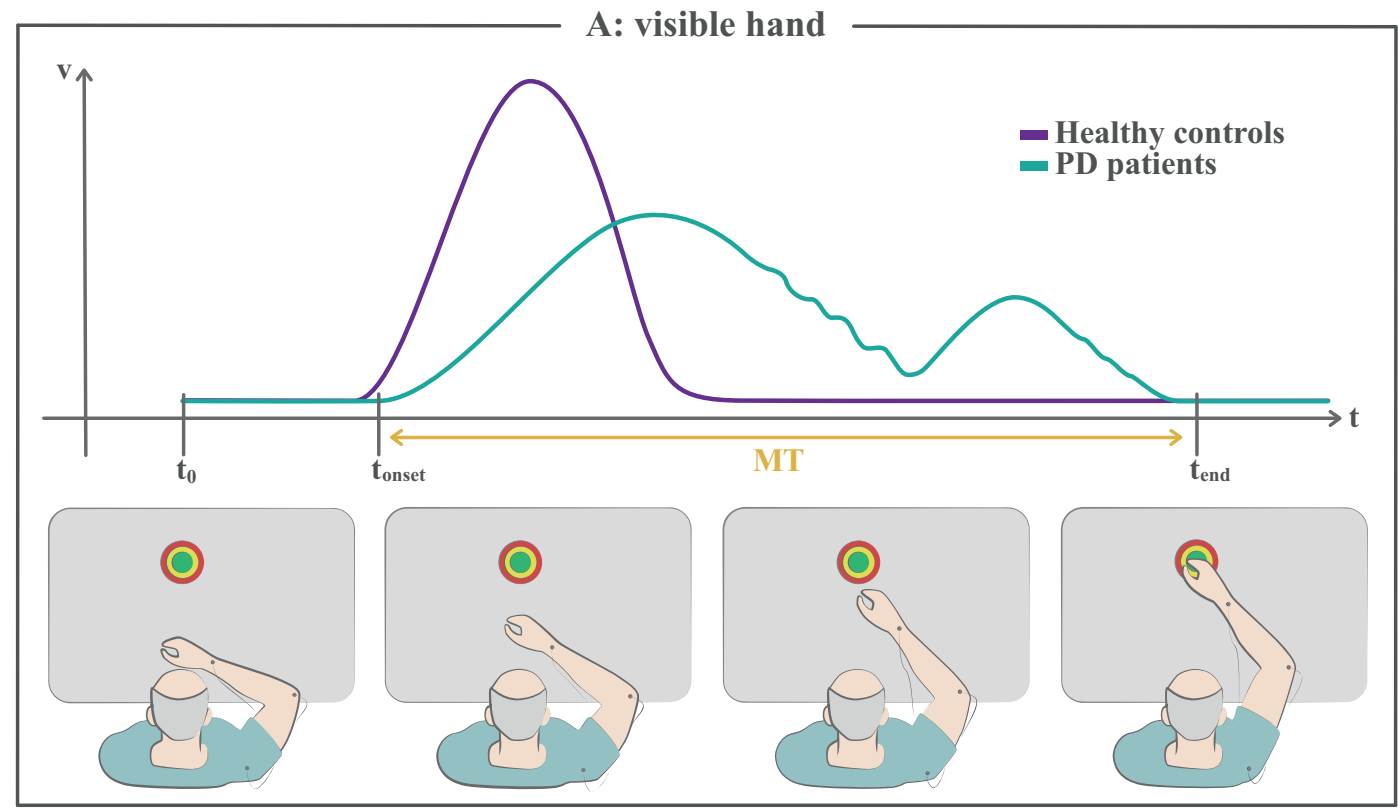

\section{B: occluded hand}

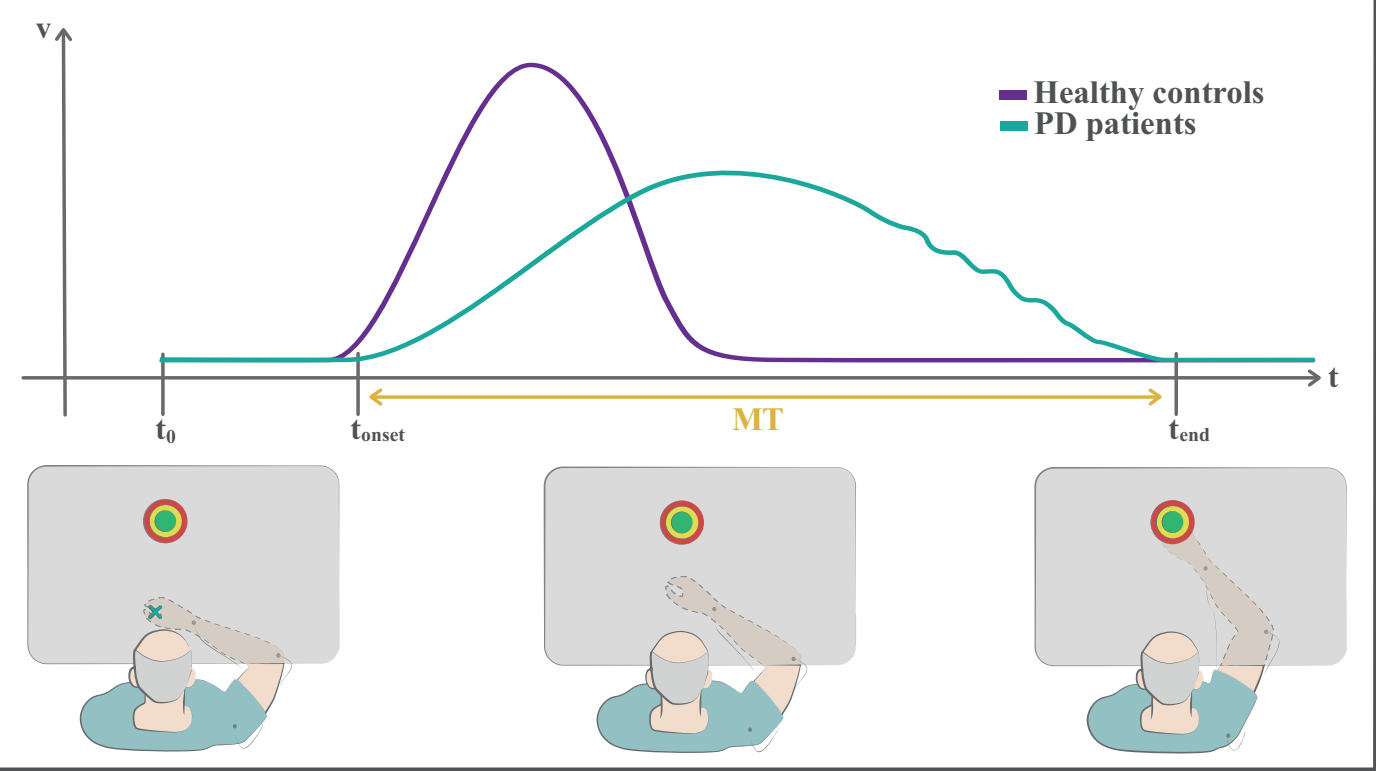

Fig. 2. Reaching tasks. A) In this kind of tasks the subject is instructed to reach a target as accurately and fast as possible, by moving planarly on a table. The bottom panels illustrate a PD patient's movement execution in progress. The top plot is a sketch depicting the corresponding velocity profile of the patient (green), in the component along the direction of movement. For comparison, the typical, bell-shaped velocity profile of a healthy subject is reported in violet. The patients usually show prolonged movement time, or bradykinesia, lower peak velocities and initial undershooting, or hypokinesia, which entails corrective submovements to reach the target. The deceleration part of the movement may be characterized by small fluctuations of velocity. B) In this version of the task, the vision of the moving hand is occluded, while the initial hand position is signaled and the target is always shown. Even though corrective submovements are still possible through proprioceptive sensory feedback, this task is generally performed ballistically in a single shot. Patients show bradykinesia and may also show hypokinesia (not illustrated here). Please, note that the actual velocity profiles, coarsely represented here and in the next figures in their main features, may differ depending on the subject and on the specific experimental conditions. MT, movement time; $t_{0}$, starting time of the task; $t_{\text {onset }}$, onset time of the movement; $\mathrm{t}_{\text {end }}$, ending time of the movement. 
first movement will undershoot the target, resulting in hypokinesia (Fig. 2A). However, Berardelli et al. [21] showed that PD patients can actually increase the size of the first burst for longer movements, thus the agonist activity is not a fundamental limitation. From these early results it was possible to draw that if patients are able to produce a movement with a normal organization but simply reduced in magnitude, the essential deficit must be in scaling the dynamic muscle force to the goal distance, i.e., there is a failure in the correct association between the perceptual cognition of the goal and the programming, or delivering, of the proper instruction to move [21-24].

\section{Distortion of the speed-accuracy tradeoff}

As Flowers and others pointed out [7, 8], if PD patients in a reaching task exploit corrective mechanisms in order not to fall short of the target point, this way lengthening the movement times, then hypokinesia can explain bradykinesia, the latter becoming a secondary phenomenon. On the other hand, not all the patients show corrective submovements [19, 25], and bradykinesia has been reported also in nonvisually guided movements [26] (see Fig. 2B) and for small target distances [27]. Now, as defined by the current criteria of the Movement Disorders Society (MDS), PD bradykinesia consists of a progressive decrement in speed or amplitude as the movement is continued [28]. In this definition, bradykinesia and hypokinesia seem to be actually combined. This is because PD patients seem unable to perform repetitive or alternating finger or hand movements of normal amplitude at normal speed, thus both signs are generally present on medical examination, although not always simultaneously [28]. Under the hypothesis of reduced muscular scaling [21], the bradykinesia and hypokinesia must be indeed seen as two facets of the same underlying pathophysiological issue. In fact, when assessing upper limb motor performances by means of clinical motor scales, such as UPDRSIII (Unified Parkinson's Disease Rating Scale, motor section [6]) and the MBRS (Modified Bradykinesia Rating Scale [29]), amplitude impairments appear to be worse and more prevalent than speed or rhythm impairments [30], even though clinical raters generally give greater weight to amplitude than to speed or rhythm [31]. Dopaminergic treatment seems to predominantly improve the bradykinesia [30], while deep brain stimulation (DBS) at the STN improves the speed and amplitude measurements, affects less the rhythm, and does not affect the decrements of speed and amplitude [32]. To note, the appearance and prevalence of one of the two signs considerably depend on the motor task and its requirements [33-35].

In two consecutive studies, Sheridan and colleagues [36, 37] demonstrated that excessive movement variability is a major determinant of bradykinesia. They suggested that hypokinesia and bradykinesia are not primary deficits, but rather a consequence of adaptation to this inherent variability. In other words, rather than being simply an inability to produce adequate forces, the parkinsonian pathological behavior can result from the fact that patients are not able to properly program these forces and thus control their degree of accuracy at normal pace, so they must trade off speed for accuracy in a dysfunctional manner, going disproportionately slow to compensate for likely endpoint errors. This can be called "compensation hypothesis" [8, 36-39].

More recently, Doan and colleagues [40] showed that accuracy constraints, imposed through the level of water fill of a glass to grasp and drink from, led to the appearance of different control strategies. In the high accuracy constraint condition, healthy subjects increased the peak acceleration and peak deceleration of reaching, as well as the time to peaks, so that movement time remained constant between conditions. Instead, PD patients off medication decreased the peak velocity, peak acceleration and peak deceleration, while increasing the duration of the planning phase, with an increased number of movement units and variability of the reach phase duration. Thus, nonmedicated PD patients did not modulate their motor response to keep the overall reach duration consistent, but instead tended to satisfy the accuracy constraint by spending more time and resources in planning to reach precisely, in line with the compensation hypothesis. The authors speculated that the increased duration observed for the premotor phase implies that the high accuracy constraint imposes greater cognitive commitment among the off-medication PD participants, also coherently with attentional interference observed in this population [41]. PD patients on medication did not show significant differences with healthy controls, nor great modification of kinematics and number or duration of movement units with the accuracy level. This indicates that dopamine replacement may not only restore movement timing, but also improve selection and execution of the initial feedforward motor plan, regardless of implicit task constraints [40]. Interestingly, the effects of dopaminergic medication on movement time and on 
peak velocity are quite persistent, while the effects on early kinematics, i.e., peak acceleration, rapidly deteriorate after drug consumption, perhaps due to a progressive decline in movement programming capabilities [42].

If bradykinesia is an active strategy to compensate for deficiencies in planning, manifested in difficulties in developing precise movement trajectories at high speed, it is reasonable to expect that PD patients show prolonged deceleration phases associated with visual guidance. Flash and colleagues [43] reported asymmetrical velocity profiles with a short initial accelerative phase, followed by a prolonged interval composed of alternating decelerative and accelerative phases, while Castiello and colleagues [44] reported a significant decrease of time spent for decelerating after dopaminergic medication. In the work of Rand et al. [45], when movement accuracy was constrained, the number of zero crossings of the acceleration curve was accentuated and the deceleration phase became longer and more variable. In contrast, Phillips and colleagues [46] reported that patients spent more time in the accelerative phase of the movement even when moving at their preferred speed, revealing inefficiencies in the rate of force production for the required accuracy, rather than increased caution and visual guidance. This leads to more submovements and zero-crossings of the acceleration function when moving at a comfortable pace, and more endpoint errors when moving at a sustained pace. Authors proposed that an altered synchronization of motor signal outflows from BG to supplementary motor area (SMA) may cause problems with maintenance and continuation of behavioral output observed in PD [46].

At this point, it is reasonable to wonder whether bradykinesia is still present when there is little requirement for movement precision. Teasdale and colleagues [33] early analyzed the temporal structure of the movement organization rather than the spatial accuracy, and showed that PD patients can actually vary the duration of their movements to comply with temporal constraints. Without any accuracy requirement, i.e., performing an initial $20^{\circ}$ elbow flexion in minimum time without stopping at that precise location, bradykinesia was still evident. However, patients increased their speed to a greater extent ( $15 \%$ for patients against $9 \%$ for controls, on average) when passing from a condition in which they should move "as fast as possible" to a condition with an imposed duration. Given also the previous results of Sanes [39] that PD patients performed as quickly and accurately as elderly subjects when the task had a low index of difficulty, Teasdale and colleagues suggested that the task requirements and the experimental context may influence PD patients' performances to a degree that was underestimated before. On the other hand, even though patients were able to modulate the time to reach the peak force when requested to go faster as well as the healthy subjects, this peak occurred later in time both absolutely and relatively to their own movement duration, as also found later in [46]. This implies that the temporal structure of movements is altered in PD and the slowness cannot be simply accounted for by a deficit in motor programming. Since slower, not faster, movements were characterized by a greater number of EMG bursts, Teasdale and colleagues proposed that, rather than problems in matching the force to the goal distance, $\mathrm{PD}$ patients may have lost ability in controlling and sustaining muscle activation due to abnormalities in recruitment of motor units [33]. Corcos et al. [47] reported that some patients off medication had a reduced ability to generate rapid extensor contractions and that measurements of muscle strength and force relaxation correlated with changes in clinical status. At least in advanced stages, PD may therefore determine a difficulty in deploying muscle force rapidly, which may represent the primary cause of the manifested bradykinesia.

\section{Bradykinesia as deficit in motor motivation}

In 2007, Mazzoni et al. [26] presented an influential study on reaching movements in PD that opened new perspectives on the discussion about bradykinesia. Patients were asked to make a certain minimum number of reaching movements within a predefined range of speed, without vision of their moving hand. Variable levels of difficulty were obtained by changing the required speed and the target distance. Patients showed movement trajectories and velocity profiles similar to controls. But, despite they were capable of moving at high speeds with preserved endpoint accuracy, patients manifested an increased sensitivity to the experimental condition difficulty, in particular the energetic demand of the task, which led them to select slower movements more often. Thus, bradykinesia seemed to be caused by a distortion in the mechanism of speed selection attributable to an abnormally perceived effort, rather than by an anomaly in the speed-accuracy trade-off. This PD patients' sensitivity to the energetic cost of the movement was depicted not as a motor weakness but as a motivational deficit. 
Authors suggested that the level of tonic dopamine from the substantia nigra to the neostriatum, which is abnormally low in PD, might signal an implicit motivation value to the motor centers, this way regulating the likelihood of spending energies to move fast. Deficiency in motor motivation would then determine a shift in the balance between the perceived reward of accomplishing the task and the energetic cost required to do it. This is in line with the models of optimal feedback control that predict movement trajectories on the basis of an optimization of cost functions [48]. Shadmehr and Krakauer [49] proposed that the BG help generate both the expected costs of motor commands, related to the energy expenditure and to the amount of noise in the produced forces (which results in variability and thus greater difficulty in controlling movements), and the expected rewards of internally predicted sensory states. This framework is also consistent with findings that pathologies of the BG affect the conversion of monetary incentives into isometric force [50], movement speed [51] and initiation times [52].

Experimental data on animals, such as mice and primates, have shown that the level of direct involvement of BG in movement execution varies among experiments and species, but BG activity always tracks and predicts kinematic parameters [53]. In particular, animal BG seem to regulate a sort of "movement gain" or "movement vigor", i.e., the rate of movement or any speed covariate, according to the context or task demands [53-56]. Moisello and colleagues [57] provided behavioral evidence on humans that is the balance between the outputs of direct and indirect pathways in BG that specifically determines a modulation of kinematics of voluntary movements. They showed that the flexibility of kinematics selection is overall reduced in BG diseases, but the pattern of impairment is opposite: when the direct pathway is affected (PD), patients select low-effort movements, or fail to elicit higher muscle force levels as normal subjects do in unpredictable target conditions, whereas when the indirect pathway is affected (Huntington's disease), patients do not exploit the option of reducing the effort in predictable target conditions, e.g., when the targets are presented in a predefined order.

The works of Mazzoni et al. [26] and Moisello et al. [57] demonstrated in humans that deficits in BG function lead to movements of reduced vigor when the subjects have to move at instructed velocity. This left open the question of whether patients, if free to choose their own appropriate speed, will still move more slowly or will adopt different control strategies. Baraduc and colleagues [27] tried to answer this question with a free choice, variableamplitude operant task, in which healthy subjects, PD patients on and PD patients off STN-DBS had to move as quickly and precisely as possible a handle towards several targets on a transversal line, without vision of their moving hand. The authors used a modelling approach based on optimal feedback control theory [48] to test if the organization of movements in the PD group was ultimately similar to that of healthy controls. Furthermore, they wanted to test if movements of off-DBS patients and those of on-DBS patients could share the same kinematic properties with just a global change in velocity levels. The endpoint variance, the peak velocity and peak acceleration and their times were all significantly affected by PD, while DBS restored the peak kinematics but not the endpoint variability. For all groups, movement time and endpoint variability increased with movement amplitude, in contrast with a minimum-variance model [58] according to which movement duration is selected as the minimum time that allows to minimize the final endpoint variance required by the task. Instead, data were coherent with a model based on the minimization of neuromuscular costs while satisfying the constraint of reaching the measured endpoint [59]: movement time was reproduced as the duration of minimum-cost movements that respected the experimentally derived dynamic range of motor commands, i.e., range from baseline to the maximum population activity in the motoneurons. This computed motor range could describe both healthy and PD subjects' trajectories and was mostly constant across movement amplitudes in all participants. The acceleration at $100 \mathrm{~ms}$ from the start of the movement was modulated by target distance for controls and patients on DBS, but not for patients off DBS, who showed a plateau. Since the initial acceleration conveys information about the motor planning and is directly proportional to the rate of rise of muscle force, these results demonstrated that the differences in performance between controls and patients were not linked to different motor control strategies but could be accounted for by an abnormally narrow motor range available for PD patients. Therefore, it seems that bradykinesia may be not due to an underestimation of the intensity of necessary impulsive force [21], but to a limitation in the mechanism that produces the motor commands. Baraduc and colleagues suggested that the narrow motor range in PD may be caused either by agonist-antagonist coactivation 
during movement, due to abnormal supraspinal regulation of spinal networks, or by a lack of central motor activation imposing an internal limit on the expendable neuronal effort. But, given that upper limb bradykinesia cannot fully be accounted for by excessive antagonist activity [24], the central limit appears to be the better candidate to explain all the reported results, and the observed changes in the available motor range among groups and with DBS/levodopa intervention can be interpreted as changes in the level of implicit motor motivation [27].

In the frame of the theory of motor motivation, PD patients would show efficient performances when the energetic cost of the movement was very low, since this condition would augment their poor motor motivation. Gepshtein and colleagues [60] tried to test this hypothesis by using an ideal planner framework as an individual benchmark of performance, that is measuring each participant's performance against his or her individual precision of movement. They engaged PD patients in a monetarily rewarded task of pointing towards targets on a monitor screen. The targets were placed above and below the starting position in order to vary the energetic cost of the movement as a consequence of gravity. Penalty regions were defined in the vicinities of the rewarding target in the direction of movement or in the orthogonal direction. The ideal planner was defined individually by modelling optimal performance, i.e., modelling the choice of the aim point that maximizes the expected net gain in the face of the estimated individual endpoint variability. When the movements were assisted by gravity, PD patients performed similarly to their optimal planner and also to age-matched controls. When the movements were against gravity, both the healthy and PD participants showed inferior scores with respect to their optimal planner, but patients' performance was significantly worse than elderly controls. These results confirmed that patients are able to plan pointing movements as effectively as control subjects, but they are more sensitive to the biomechanical cost of the movement. Therefore, the energetic cost of action sets a limit for efficient planning and this effect is mediated by tonic levels of dopamine in the dorsal striatum, which determine a threshold of energetic motor cost beyond which it is not possible to optimize individual motor behavior [60].

\section{Kinesia paradoxa and effect of sensory cues}

An interesting aspect of movement execution is that PD patients can exceed their response speed in the context of urgent situations, so much as restoring their performances [61]. This phenomenon is called "kinesia paradoxa" and may be seen in line with the existence of an implicit motor motivation. Indeed, Majsak and colleagues [62] demonstrated that PD patients are able to exceed their self-determined maximal speed of reaching when asked to reach and grasp a ball in motion, which acted as a pressing, externally timed cue. This enhancement of performance with respect to the stationary condition came with preserved accuracy. Therefore, PD patients do not seem to have lost ability in moving fast overall. Their slowness appears to result more from an inability to internally maximize the movement speed, or from a distorted speed selection on a motivational basis [26], rather than a compensation for hazard. Now, the contextual variation of movement speed may be a general property of the motor system, being also present in healthy subjects [63]. This means that the normal speed of reaching may be determined not only by the accuracy constraint of the task but also by the internal motivation, and that modulation of movement time can overcome usual constraints in a situation of urgency [9].

In a successive study of Majsak and colleagues [64], PD patients slowed down and reduced the grasp components (slower velocity of hand opening and closing, smaller maximal aperture, longer time to maximal aperture) in both stationary and moving ball conditions, while the visual motion cues and external temporal constraints had a greater beneficial effect on performances of reaching than on grasping. This suggests that bradykinesia might be a possible compensation for poor prehensile abilities. However, the phenomenon of kinesia paradoxa has been documented also for tasks that do not require fine motor control [61]. Bieńkiewicz and colleagues [65] were able to study the ability of PD patients to catch a moving object through a metal disk attached to the hand, thus removing the prehensile component. Also, they let the temporal dynamics of the imminent task be uncertain and not predefined, thus participants could not fully prepare the subsequent action in advance and use the cueing for timing the initiation, but mostly rely on online movement control. Not only the ability of PD patients to successfully intercept the object was preserved, but also the kinematics of their reaching was adapted to the speed of the moving ball in different cueing situations, demonstrating the intact effect of urgency on the performances of patients.

Paradoxical movements can be related to the general, beneficial effect of sensory stimuli on PD 
patients' performances, especially the visual ones [66-68]. Glickstein and Stein [61] noted that the stimuli that often elicit such paradoxical movements are indeed similar to those that relay visual information through the pontine nuclei to the cerebellum for visuomotor control. In a behavioral study, Schenk et al. [69] found a much smaller GPi-DBS effect on the kinematics of externally rather than internally guided movements in PD patients involved in a standard prehension task as well as in a grasp-only task. These results confirmed the limited involvement of BG in the control of externally timed movements. In particular, as clarified below in "Abnormal movement-related brain activity", this might be due to the fact that movements driven by external cues are primarily controlled by lateral circuits involving the premotor cortex, which are less affected by the PD. This can account for the clinical efficacy of external stimuli in improving movement freezing [70]. In [71], Bieńkiewicz and colleagues used a travelling light display reproducing the real hand motion of a healthy subject as a kinematic guide for the patient. They observed that participants moved significantly faster when the same movement was triggered by the display compared to the self-paced condition, i.e., moving as fast as possible without a guide, and the speed of the display had a significant effect on the movement time. This is in line with the results of Majsak et al. [62] and the others concerning kinesia paradoxa. Bieńkiewicz and colleagues [65] suggested that such improvements in the motor behavior are modulated by changes in the dynamics of the ongoing sensory information: the temporal information of external sensory cues may help bypass the damaged habitual control loops in the BG (caudal putamen) and allow a direct involvement of the preserved goal-directed control ones (STN, GPe) [72], thus enhancing the motor performances. Bek and colleagues [73] recently demonstrated that, in PD patients as well as healthy subjects, movement duration can be modulated to a greater extent by adding to a simple visual cue a moving human hand to imitate. This was explained as a major focus on the dynamics rather than on simply the endpoint, when the kinematic information of the hand was available. Furthermore, imitation of trajectory and visual tracking improved when following a human hand (biological stimulus), compared to following a moving shape (non-biological stimulus) with the same kinematic profile. However, in the work of Castiello et al. [74] significant facilitation effects by human action observation took place for PD patients only when the action matched their actual performing capacities, giving hints as to BG involvement in representation of observed actions.

\section{Hypokinesia and defective proprioception-based control}

During non-visually guided arm movements, PD patients tend to make larger spatial errors than healthy controls $[8,25,43,75]$. This can be related to the impossibility to exploit corrections, even though secondary trajectory adjustments are possible also in absence of visual feedback, by relying on proprioceptive sense [76, 77]. Klockgether and Dichgans [25] reported that even though patients showed consistent undershooting when the vision of the hand was occluded, their movements were both fast and accurate when the target disappeared after the starting signal. Thus, that population of patients could correctly perceive and memorize target position and potentially use this information to successfully program the movement. Indeed, the spatial working memory (or visuospatial mental imagery) seems to be intact in patients performing three-dimensional pointing to remembered target location in the dark [78], even though the degree of hypometria is generally greater in memory-guided movements [79].

If there is not a basic motor planning deficit causing hypokinesia, then it is plausible that the shortfall is in the afferent signal from the periphery and that the extreme reliance on visually-based compensatory mechanisms is the consequence of proprioceptive deficits [75, 80]. Interestingly, PD patients show reduced sensitivity for detecting changes in limb position both on proximal [81] and distal joints [82]. They also need significantly larger limb displacements to detect passive joint motion [83]. These kinesthetic deficiencies often correlate with disease severity and duration [81], but they can appear very early and even precede the motor signs (for a review, see [84]). However, microneurographic recordings of muscle spindles [85] and experiments on illusion of limb displacement by muscle vibration [86] have shown that if proprioception is disturbed in PD, it is not due to peripheral malfunctions. Therefore, an alternative explanation is an anomalous central processing of this afferent signal and hypokinesia may result from an abnormal sensorimotor integration $[3,25$, 87-90]. Indeed, PD patients seem to be selectively impaired in conditions that require integration of arm proprioceptive feedback with visual (or spatial 
memory) information for achieving accurate movements [90]. Their accuracy of reaching seems not to degrade when the starting location of the hand is visible, thus minimizing the so-called "localization bias" [91], while dramatic endpoint errors are likely when starting in the dark $[8,25]$. These deficits in the processing of proprioceptive information may occur already at very early stages of PD, while those in the use of visual feedback may develop progressively in later stages [92]. Furthermore, when vision is occluded, a reduced spatio-temporal coupling between the pointing component and the postural adjustments component (control over center-of-mass displacements) emerges in PD [93], revealing an involvement of BG in the integration of proprioceptive information for posturemovement coordination.

Another interesting occurrence is that PD patients usually speak in a low voice, thinking they are speaking normally $[94,95]$, just as well as they think they are matching the target in goal-directed arm movements when they are actually undershooting [25]. In [87], Moore proposed that during slow, closed-loop arm movements the corollary discharges (efferent copies) that the brain uses to internally estimate the amount of force produced are diminished in PD with respect to the kinesthetic, i.e., proprioceptive, feedback from the periphery. This mismatch would cause an overestimation of the distance covered by the arm and induce the motor centers to dampen the commands towards the musculoskeletal system, resulting in hypokinetic movements. However, in a study by Klockgether et al. [96] PD patients were systematically hypometric with respect to control subjects also in passive movements, not involving internal corollary discharges. This result can be accounted for by an altered processing of kinesthetic information in the BG-related networks [90, 96]. Demirci et al. [88] found that PD patients underestimate more than healthy subjects the motion of their fingers during passive displacement, in contrast with Moore's model and with the previous findings of overestimated hand movements [8, 25, 96, 97]. Authors suggested that kinesthesia is reduced in PD, which can account for the underestimation, but also the corollary discharges are diminished, so that the error signal is nil and the final motor output is always decreased with respect to the task requirements. This "tuned-down" sensorimotor apparatus explains the discrepancy between the impression of correct motion that patients have and their actual performances, which is reset when exteroceptive information is available [88].
Online modulation of movement trajectory has indeed appeared to be influenced by BG activity [98, 99]. When initial proprioceptive errors are imposed through force perturbations, BG-damaged patients seem not to be able to adjust their ongoing arm movements effectively and smoothly, which suggests an involvement of $\mathrm{BG}$ in the online feedback control $[99,100]$. However, in experiments of Desmurget and colleagues [101] no substantial deficit was found in PD patients when asked to accurately point towards a target whose location jumped during ocular saccade, i.e., when vision is suppressed (double-step reaching task). In this case, all subjects responded with a smooth adjustment of the trajectory to reach the new location, indicating an effective, subtle modulation of the ongoing motor command. The only important difference between groups was the delay with which patients reacted to the target jump with respect to healthy subjects. Instead, the authors found an abnormal result in the PD group when participants were asked to point towards targets jumping at the individual movement onset, thus requiring them to exploit discrete corrective submovements. PD patients were dramatically late or even unable to employ a secondary adjustment before completing the initial response. Most of them did not show the overlapping of subcomponents typically seen in the velocity profiles of healthy subjects $[102,103]$. These results revealed, first, that BG may not be critically involved in non-visual feedback loops involving a smooth modulation of the ongoing motor command (see also [104, 105]). Interestingly, dopamine medication did not consistently normalize performances to the level of controls in several proprioception-based tasks [75, 81, 106-108], suggesting that disruption of the dopaminergic circuits may be not, at least primarily, responsible for all the proprioceptive processing deficits of PD patients. Second, from the work of Desmurget and colleagues [101] it emerges that the BG circuits can instead importantly contribute to the generation of discrete corrective submovements, as also shown in sequential and simultaneous movement tasks [109-113], especially in situations where the movement sequence is not known in advance [114]. Given that the motor plant is non-linear, in order to produce successive movements the brain must estimate the real time position and velocity of the limb by deploying a forward model of the arm dynamics [115]. Therefore, the reported deficits on iterative online control suggest that the BG are involved in forward modelling [101], or they may have a key role in a sort of contextual motor decision making, controlling 
the timing and the necessity of corrective submovements [116], which is coherent with the concept of BG as a "gate" for action release [117].

It is important to note, however, that gross noncorrective submovements, i.e., submovements not performed to increase accuracy and corresponding to reversal in the trajectory, can emerge in adults in the process of motion termination, i.e., during final antagonist co-activation near the target. Instead, fine non-corrective submovements, corresponding to zero-crossing of the acceleration or the jerk profiles, may be seen as fluctuations in movement velocity emerging from mechanical and neural sources of motion variability [118]. When accuracy constraints are more stringent, these trajectory irregularities are more pronounced. Indeed, the decreasing of speed due to the speed-accuracy tradeoff may come along with a less smooth trajectory, because maintaining low velocities requires a fine regulation of motor unit activations for the steady production of muscle force [119], complicated by aging effects on motor output variability [120]. The presence of such submovements was demonstrated also in the PD population [121], but a specific deficiency was shown in smooth motion termination, also coherently with findings of BG involvement in movement deceleration and stopping [122]. PD patients produced, instead, fine submovements at least as frequently as controls: the majority of their submovements were not corrective but rather due to irregular fluctuations of velocity [121], perhaps exacerbated by the mentioned anomalies in muscular activation [23, 123, 124], especially at low speeds [33].

The fact that conscious perception of target jump interferes with automatic motor control in PD [101] has been recently contested in [125]. Indeed, when the target jump is imposed at the movement onset, the akinesia in limb motion inevitably prolongs the period from target appearance to target displacement. This may disproportionately impact the performances of patients, since their long preparations do not favor quick re-modulation of the trajectory [126]. By using large target displacements during initial saccadic fixation to target, but still consciously perceived by the subjects, Merritt et al. [125] ensured equivalent onsets of target displacement for PD and controls and demonstrated that automatic trajectory modifications were equivalent in both groups and were unaffected by dopaminergic therapy. This questioned the idea that BG actually mediate the online motor control. Interestingly, the PD group did not increase the movement duration in jump trials with respect to stationary trials as much as age-matched healthy controls did. This suggested that aging-related deficiencies in online motor control may be masked by PD-related dysfunctions of dorsal striatum in suppressing automaticity of behavioral responses (i.e., inhibitory control).

The magnitude and variance of perceptual errors about body configuration depend on movement duration: in healthy adults, longer movements result in a stabilization of these errors, because of a more accurate and certain estimate of hand position when the duration increases [127]. Koop et al. [89] found that, instead, PD patients' perceptual errors peaked at longer times than controls and did not significantly decrease for prolonged movements, while their variance continued to increase with duration. The absence of a stabilization of the perceptual errors informed authors of an abnormal proprioceptive feedback and/or an abnormal internal predictive model. Their proposal depicted hypokinesia as an underestimation of the amplitude of movement needed and may be seen in line with the theory of inadequate scaling of the motor commands to the effort required by the task [21]. Now, when the confounding effect of initial hand localization bias is removed from the error measurement, the movement extent error and variability can still result increased in patients with respect to control subjects, without a concomitant increase in direction error and variability ([25, 128], but see [43]). Desmurget and colleagues [129] demonstrated that behavioral tasks requiring amplitude planning produce increased activation in the BG network. Changes in the BG activity as a function of the movement extent and/or velocity have been observed in other studies [130, 131]. This suggests that BG participate in the planning of movement amplitude (or its covariates, such as effort), by modulating cortical activity that prepares the instruction to move and/or by appropriate peri-movement tuning of the spinal interneuron systems [128, 129], as also suggested by Baraduc et al. [27].

Hypokinesia may be hence a result of a deficit in pre-movement planning, independent of proprioceptive online processing. Behavioral data have indeed suggested that the estimation of the current hand location during reaching movements is mainly based on the efferent signal and little on proprioceptive feedback [104]. On the other hand, as we have seen, the vast majority of studies showed that PD patients have poor accuracy only when they have to rely on proprioceptive information. Mongeon and colleagues [80] investigated whether this inaccuracy depends on an 
impaired sensory processing in the phase of movement planning or in the phase of online movement control. Subjects were asked to point in the 3D space towards visually memorized targets, with or without vision of the arm, and targets defined proprioceptively by active or passive reference movements. There were no significant differences in the movement duration, magnitude and time-to-peak velocity between groups (controls, PD on, PD off medication state). Final spatial errors were larger in patients than in controls only for proprioceptively-defined targets, especially for medicated patients. While initial spatial distances between hand position at $150 \mathrm{~ms}$ and target position were similar, the percentage of change in hand position relative to the target between time of peak velocity and time of movement endpoint was lower for patients, i.e., they compensated for spatial distance relatively less during the deceleration phase. This happened mostly in proprioceptive-based movements. More specifically, PD patients' hand position at peak velocity was systematically higher on the vertical axis, indicating that they tended to first raise the hand at the elevation of the target in the acceleration phase and then reach out in the target's direction in the deceleration phase. This strategy could have been employed to simplify the control of the multijoint movement, as a compensation for their proprioceptive deficits. Taken together, these results suggest that the PD patients' lower endpoint accuracy cannot be primarily accounted for by a different planning strategy as much as by an ineffective movement guidance in conditions of absence of visual feedback. As the authors pointed out, since the results on the sensorimotor integration in PD are somewhat divergent, the contribution of $\mathrm{BG}$ in the online control of movements might be context-dependent. Consistent with this view, neurophysiological studies in humans have shown that BG activity can be correlated with the number of corrective submovements only during the execution of a motor task in which a proprioceptive error is induced, but not when visually guided online control is required [132]. Thus, as mentioned above, BG may be selectively involved in submovement generation mechanisms based on proprioceptive information. Coherently, proprioceptive localization errors can be reduced with STN-DBS, even though at the cost of increased endpoint variability [133].

Even in full-vision condition, PD patients' performance becomes fully accurate only when both the target and the hand are simultaneously visible [134]. If PD patients extremely rely on visual guidance to compensate for impaired proprioceptive integration, then their gaze should be persistently aligned with their hand in pointing towards objects. Lukos and colleagues [135] observed that during the first phase of a full vision reach-to-grasp the eye-hand positions correlation was higher in PD patients and the minimum horizontal and vertical distances separating the gaze position and hand position on the screen were significantly smaller compared to controls. Patients also made numerous short saccades between the thumb and the index finger during the movement, demonstrating that they almost tracked their hands with their gaze. Even when the vision of the hand was blocked, patients continued to look at the screen in a similar fashion. All these results suggest an abnormal eyehand coordination, i.e., patients over-rely on visual feedback of their hands instead of employing feedforward anticipatory mechanisms. Prediction of the movement outcome for anticipatory control may be deterred by the greater variability in the initial force impulse [136].

\section{ANOMALIES IN MOTOR PREPARATION AND INITIATION}

We have so far discussed difficulties in the execution phase of the movements but, as Flowers and colleagues noted in early studies [7, 8], patients with PD have difficulties in initiating pointing tasks, even when minimal programming is required [36]. This akinesia can be naively thought of as an extremization of bradykinesia. However, the severity of initiation delay does not always correlate with the severity of movement slowing [137]. The fundamental issue is to understand whether akinesia reflects problems in releasing motor commands, or in properly programming them [24]. In fact, planning and execution cannot be disentangled clearly when the task is complex, or when the movement entails online modulations. Therefore, motor preparation abnormalities in PD have been historically studied in delay or reaction time tasks, examining the behavior and the brain activity before the movement onset.

A reaction time task (Fig. 3) requires the participant to perform a simple movement as soon as an imperative stimulus (or "GO" signal), such as an auditory tone or a LED light, is detected. The time between the occurrence of the stimulus and the movement onset is called "reaction time" (RT) and is usually $300-500 \mathrm{~ms}$ for healthy subjects in easy tasks, such as button pressing. RT tasks can be simple 


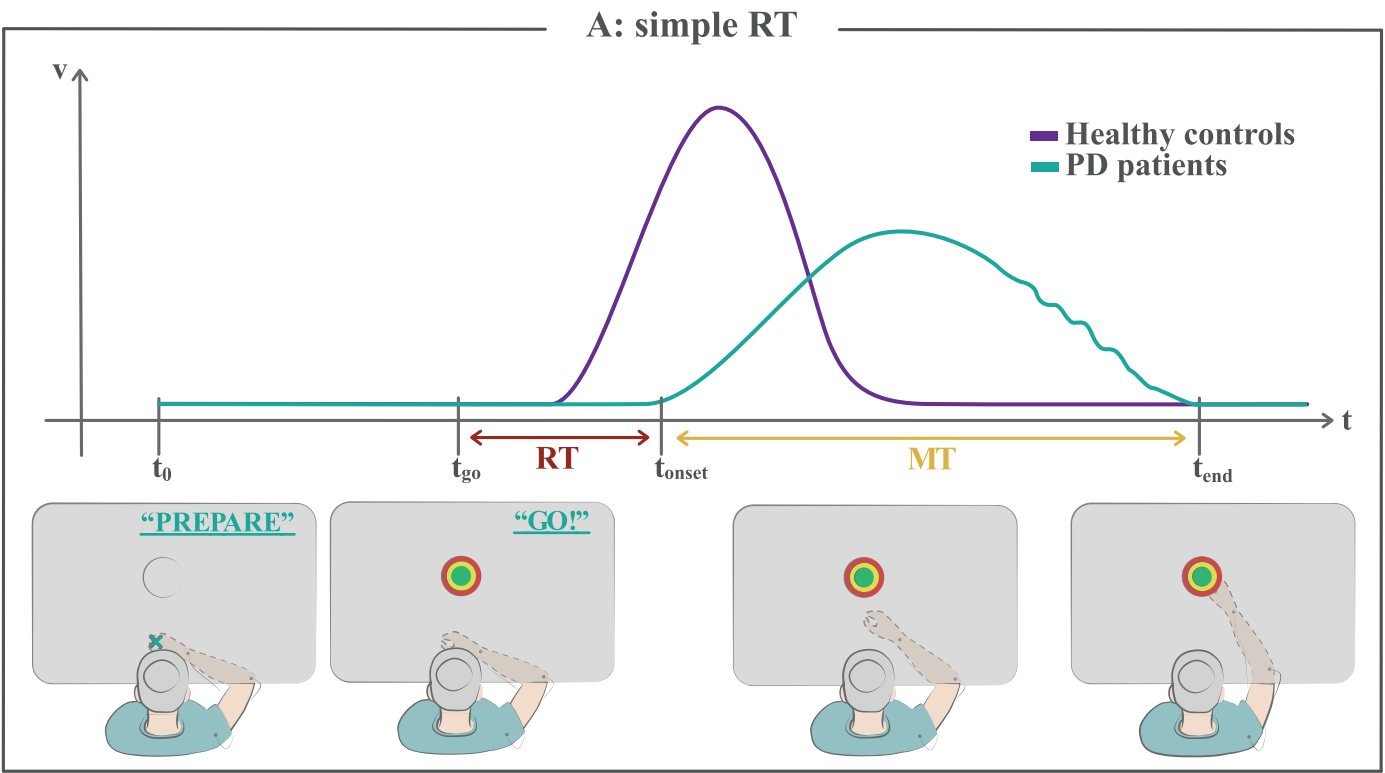

\section{B: choice RT}

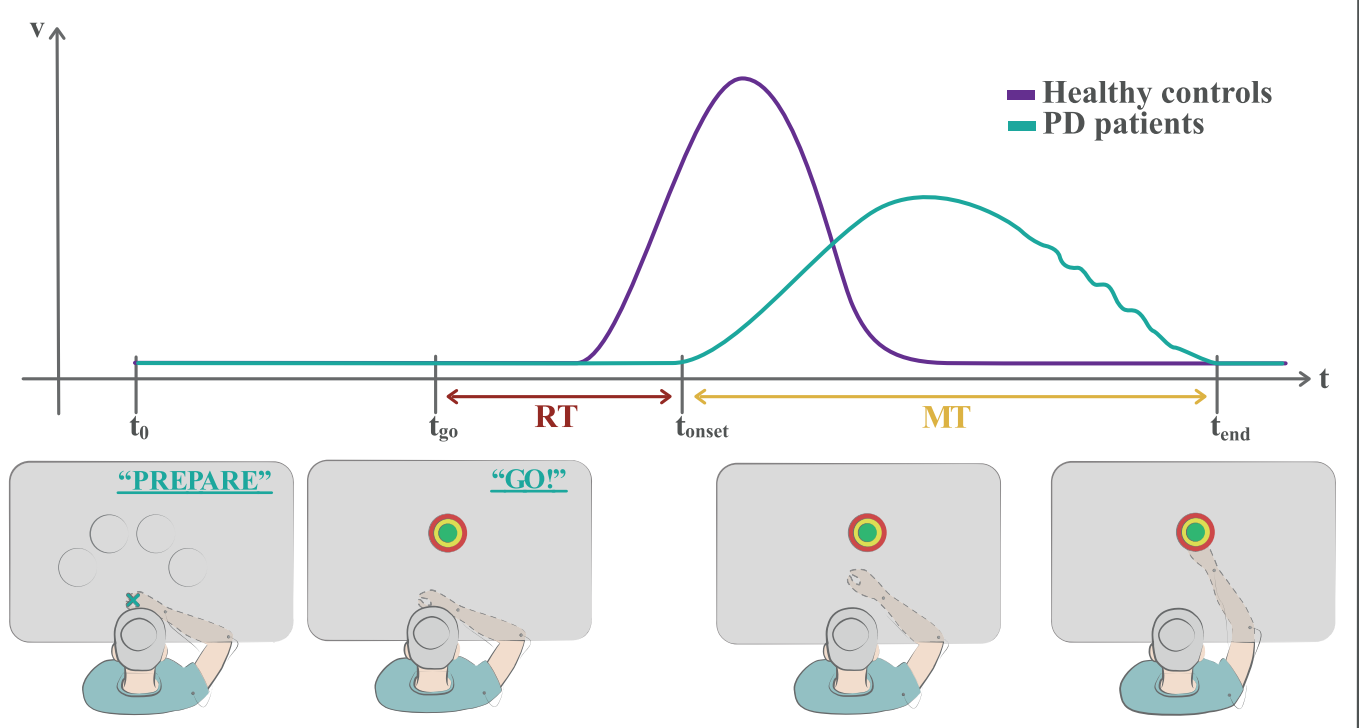

Fig. 3. Reaction time tasks. A) In a simple reaction time task, the subject knows the target position in advance, but he/she is instructed to wait for a "go" signal (imperative stimulus) to start the movement. A reaction time (RT) is measured as the temporal interval between the instant of the "go" signal appearance and the onset time of the movement. The portrayed woman with PD shows a typical delay in RT with respect to healthy controls (akinesia). B) In a choice reaction time task, some clues are given to the subject as potential responses, but the correct one is revealed only after the "go" signal appearance, so that he/she cannot fully prepare the movement in advance. The RT is generally prolonged for both healthy controls and PD patients with respect to simple RT tasks. See the text for detailed discussion. MT, movement time; RT, reaction time; $\mathrm{t}_{0}$, starting time of the task; $\mathrm{t}_{\mathrm{go}}$, time of the "go" signal appearance; $\mathrm{t}_{\mathrm{onset}}$, onset time of the movement; $\mathrm{t}_{\mathrm{end}}$, ending time of the movement.

or choice tasks. In a simple RT task (Fig. 3A) the correct response is known in advance, since it is the same on every trial, so that the subject can fully preprogramme it. Early studies consistently showed that PD patients have slower RT with respect to controls in this kind of tasks [137-139] and this result was confirmed in all the following works. This increase in RT amounts to 10-30\% [137]. While the most straightforward interpretation of this slowness is that PD affects the act of initiating a movement, it is also plausible that the impairment lies in the preprogramming of the response before the stimulus onset. In 
choice RT tasks (Fig. 3B), the nature of the response is not known in advance, so that a certain amount of response uncertainty is left before the imperative stimulus. A set of cues is involved in the task, each of which corresponds to a specific response. The required response is revealed only after the imperative stimulus presentation, then the subject can program (or complete most of the preparation) and initiate the movement.

Some early studies showed a selective impairment of PD patients in simple RT tasks and not in choice RT tasks [36, 137, 140], suggesting that the major factor determining akinesia in RT tasks is the failure in using advance information to program the response before the "go" signal. However, some other studies reported also longer than normal choice RTs [138, 141, 142], which instead suggests a slowness in the common stage of response initiation. Then, the additional slowing in choice RT tasks revealed in [138, 141] indicates deficits in stages of processing unique to choice tasks, e.g., stimulus identification, decoding of the stimulus-response mapping, response selection. These controversies in the RT studies may in fact arise from the high dependence of the response choice on the design of the task: PD patients may handle the compatibility between stimulus and response in a different way with respect to normal subjects [141]. Medication state can be a determining factor as well [143]. Furthermore, Parkinsonian subjects seem to have fundamental slowness in information processing, even when on medication, as supported by studies on "inspection time", in which the response variable is the minimum time necessary for the subject to reliably identify physical properties of a stimulus [144]. It is not clear yet how much of the RT slowness is associated with such cognitive impairments.

Recently, Carlsen et al. [145] demonstrated that the presentation of a startling acoustic stimulus not only leads to a large decrease in latency of accurate response of PD patients (as also observed in [146]), meaning that the intended action was fully prepared and ready to be initiated, but it also leads to a normalization of their movement speed, whether on or off medications. Authors thus proposed that, since patients are completely able to program the response in advance, PD affects response initiation mechanisms. Intriguingly, Haith et al. [147] found, in healthy subjects, a mean delay of about $80 \mathrm{~ms}$ between the time at which movements became appropriately prepared (enough accurate) and the time at which they were actually initiated (when free to do it). When pressured to react faster, subjects significantly reduced their RTs. Haith and colleagues were thus able to demonstrate that preparation and initiation are distinct and independent processes, and RT may be due to voluntarily delay the initiation to avoid errors. This suggests that variability in RTs between populations or across tasks can be attributed to differences in initiation rather than preparation. In this view, just as bradykinesia reflects a compensation in execution, the PD akinesia may be due to a trade-off with accuracy, abnormally prolonging the RT to reduce risk of errors. In this context, in the study of Mirabella et al. [148] healthy participants increased their RTs and decreased their MTs in reaching trials in which they knew that a stop signal could have been randomly presented during their RT period (proactive control), with respect to the trials in which they had to execute the same reach as quickly as possible without stop signals. This means that subjects used their RT to carefully process movement parameters, prior to the occurrence of the cognitively demanding event. Instead, PD patients have shown poor inhibitory control in "go/no-go" or stop-signal RT tasks (for a meta-analysis, [149]), which means that they not only have problems in initiating a motor action, but also in suppressing the already initiated ones. When an unexpected change of pre-planned action was needed to reach the correct target (reactive control), PD patients exhibited a normal increase in RTs but an abnormal increase in movement slowness and variability [150] (coherently with the results on jump trials reported above). This suggests that their capability of modifying motor plans during the initiation time is limited and they need to complete this adjustment during the execution phase.

In akinetic subjects, attentional processes may be less efficient as well, since patients with longer reaction times showed less neuronal desynchronization over the occipital areas and also performed more poorly in the cognitive tasks [151]. Therefore, impairment in the process of directing attention to a specific stimulus may be involved in the genesis of akinesia [152]. However, when asked to consciously use an attentional strategy to focus on internally timed responses rather than relying on external cues, PD patients showed significantly faster reaction times in [153].

\section{Abnormal movement-related brain activity}

To which extent can we relate the aforementioned behavioral dysfunctions to pathological neural 
activity? A disproportionate inhibition signal from the BG to the thalamus may cause a failure to reach cortico-thalamic activation thresholds to initiate the prepared motor program, which may largely contribute to the slowness observed in movement initiation and execution of PD patients [145]. Indeed, while the transmission of the motor commands from the cortex to downstream motor centers appears to be intact in PD [154], the pre-movement cortical excitability increases more slowly than normal [155]. This slowness in reaching motor excitability thresholds has been demonstrated in EEG studies measuring the so-called Bereitschaftspotential (or "readiness" potential) that rises over a widespread area of the scalp just before the onset of voluntary movements, thus reflecting preparatory activity. Libet et al. [156] proposed that the early component of this potential, which starts about 1 or $2 \mathrm{~s}$ before the EMG-detected movement onset, reflects the preparation to act soon, while the late component, with an onset of about $500 \mathrm{~ms}$ before movement, is associated with an endogenous urge or an intention to act. While this latter component is significantly higher for the self-initiated than externally triggered (e.g., via visual or auditory cues) movements, the former one does not differ between the two types of movement [157]. Dick et al. [2] showed that in PD patients off medication this premotor potential has a normal peak amplitude, but it is reduced $650 \mathrm{~ms}$ before the peak, that is 1-2 seconds before movement, especially in midline electrodes. As a net effect, the rise of the readiness potential results steeper in the PD population, even though attentional strategies may help increase early-stage activity [153]. In successive studies [153, 157, 158], with respect to normal subjects, PD patients showed a general under-activation of the medial cortical motor circuits (e.g., linking BG to SMA) for self-initiated hand movements, and an extra-activation of the dorsolateral areas (e.g., premotor area and dorsolateral prefrontal cortex) when external cues were given. The inadequate BG activation of the SMA accounts for the reduction of the early EEG component, which can be related to impairment in the preparation of proper movement commands [156]. Interestingly, in healthy subjects the readiness potential is much larger when performing a choice rather than a simple RT task [159], which can be modelled considering an extra-activation of the SMA to perform additional processing necessary for the choice, a mechanism probably lacking in PD patients [160]. Indeed, Filipovic et al. [161] found that PD patients with smaller difference in response latency between choice RT and simple RT tasks have smaller amplitudes of the readiness potential, presumably caused by reduced movement pre-programming capacity. Furthermore, the medial circuit has been associated with the ability to use predictive models to internally program action sequences with or without cues [158, 162], an ability impaired in PD [109, 158]. Instead, the patients' overactivation in the later component of the potential, nearer to the movement onset, reflects compensatory activity of the lateral premotor and parietal areas [2]. Hyperactivation of premotor and cerebellar areas for defective BG was confirmed by successive fMRI studies [163-167].

The parkinsonian slow build-up of the motor excitability reflects a delay from the preparation of the motor plan to its expression in the motor cortical areas, which delays movement initiation. Coherently, event-related desynchronization patterns in the alpha and beta bands preceding voluntary movements are abnormal in PD. In particular, the appearance of the uncoupling of population activity, necessary for movement initiation, is significantly delayed, at least for the contralateral hemisphere, revealing a programming deficit that accounts for the akinetic and bradykinetic behaviors [168-171]. Also, longer and higher beta bursts have been recently reported [172]. Torrecillos et al. [173] measured the local field potentials (LFP) from the STN of PD patients under medication and executing visually-cued reaching task with a joystick. At single trial level, within-subject correlations between pre-movement beta bursts and their movement performance revealed that, according to their timing and amplitude, these bursts can reduce the speed of the forthcoming movement and prolong the RT. In particular, the trials in which a beta burst in the contralateral STN occurred roughly $650-500 \mathrm{~ms}$ before the movement onset were associated with a reduced peak velocity and this effect was related only to the burst amplitude and not to the mean beta power in each trial. Moreover, the presence of the burst in a 200 ms window after the "go" cue was associated with increased RT. In a recent LFP recording study, Tinkhauser and colleagues [174] added that slowest movements appear to be those that are preceded by multiple bursts of beta activity occurring at short time intervals within a trial and spatially overlapping between the two STNs.

These electrophysiological studies shed new light on the involvement of BG nuclei in reaching and grasping. BG seem to release the cortex from idling rhythms in beta frequencies and let necessary 
motor channels be favored and become coherent at higher "functional" frequencies to achieve a specific movement [175, 176]. Indeed, Brown et al. [177] revealed that populations of neurons in the subthalamic nucleus and internal pallidum are coupled at $20-30 \mathrm{~Hz}$ in the off-medication state, whereas in the on-medication state they oscillate at $60-70 \mathrm{~Hz}$. Power attenuation of the alpha and beta rhythms can indeed be restored with dopaminergic medication, an effect that correlates with improvements in bradykinesia [178]. But dopamine deficiency in PD apparently results not only in excessive oscillations but also increased inter-regional coherence at beta band frequencies [179], suggesting a role of striatal dopamine in preserving subcortical structures from excessive cortical entrapment and cross hemispheric coupling that would prevent fine tuning of movements [180].

The oscillatory synchronization in the STN is then modulated by context and task demands [181]. In particular, this nucleus seems to play a selective role in response inhibition. Kühn and colleagues [170] recorded the LFP of the STN in PD patients involved in RT tasks in which an imperative cue signaled whether to move or not to move. In "no-go" trials the pre-movement desynchronization was reduced with respect to the "go" trials. In the late post-movement phase, they found an increase in beta power, i.e., post-movement synchronization, that was higher and a few hundred milliseconds shorter in latency for the "no-go" trials than the "go" trials. Thus, the STN is involved in the preparation also of externallypaced movements, other than self-paced movements, and the degree of synchronization in its beta band activity is associated with the suppression or facilitation of movement programming and initiation. Coherently, DBS of STN can partially restore the motor strategy preparation and the inhibitory control in PD patients [182]. Furthermore, other LFP recordings in PD patients revealed that ballistic fast movements of the elbow joint cause synchronized STN activity in the alpha range [183], and that also changes in alpha band oscillatory patterns can be associated with reactive control [184]. Alterations in the oscillatory activity of the BG in PD also affect the gamma and high frequency bands (for a review, see [185]). Bradykinesia of the upper limb can be indeed predicted from the beta and gamma activities of the primary motor and premotor cortices, and the STN [32]. In the paper by Joundi et al. [186] a synchronization in the gamma range in PD patients' STN developed during the reaching movement and was especially pronounced in response to cues signaling an upcoming fast reach than to cues indicating slow or normal reaches. Instead, only the timing of betaband desynchronization depended on task demands, and not its extent. This suggests functionally segregated roles for beta and gamma frequencies in motor preparation and execution.

\section{ANOMALIES IN THE COORDINATION OF THE REACH-TO-GRASP}

In studies on the reach-to-grasp task (see Fig. 4) PD patients exhibited deficits both in intensive and in coordinative aspects of movement [134,187-189]. The former regarded a generalized slowness in execution and consistently smaller hand apertures. The latter regarded a reduced capability of precisely timing the transport of joint segments, especially under high accuracy requirements, or of integrating proprioceptive information about the arm position with visual target information to control hand preshaping (i.e., distance along the trajectory between the peak speed point and the peak aperture point) in a predictive manner. In these studies, dopaminergic therapy significantly improved only the intensive aspects of the movements, suggesting that coordination must not strictly rely on tonic levels of nigrostriatal dopamine, i.e., on a regulation of the gain of the motor circuit, but rather on a choral pattern of wide-ranging cortical activities and on the role played by BG of integration of such activities, supporting sensorimotor processing.

Even if both reaching and grasping are slowed down in PD, the different phases of this movement may be in fact differentially affected by the disease [190, 191]. This differential impact may be related to a distorted programming of specific movement parameters or impaired online visuomotor control, and leads to a breakdown of the harmonic coordination between the components of the reach-to-grasp [134]. These deficits in planning or sensorimotor processing should worsen as the disease evolves and improve with medication. The experimental study of Negrotti and colleagues [190] was designed to clarify the effect of disease stage and L-dopa on kinematic control of the reach-to-grasp. For this purpose, they enrolled healthy controls, PD patients in early stage and drug-naive, and PD patients in advanced stage and either in "on" or in "off" state of medication. The task comprised targets of different sizes and placed at different distances, in different 

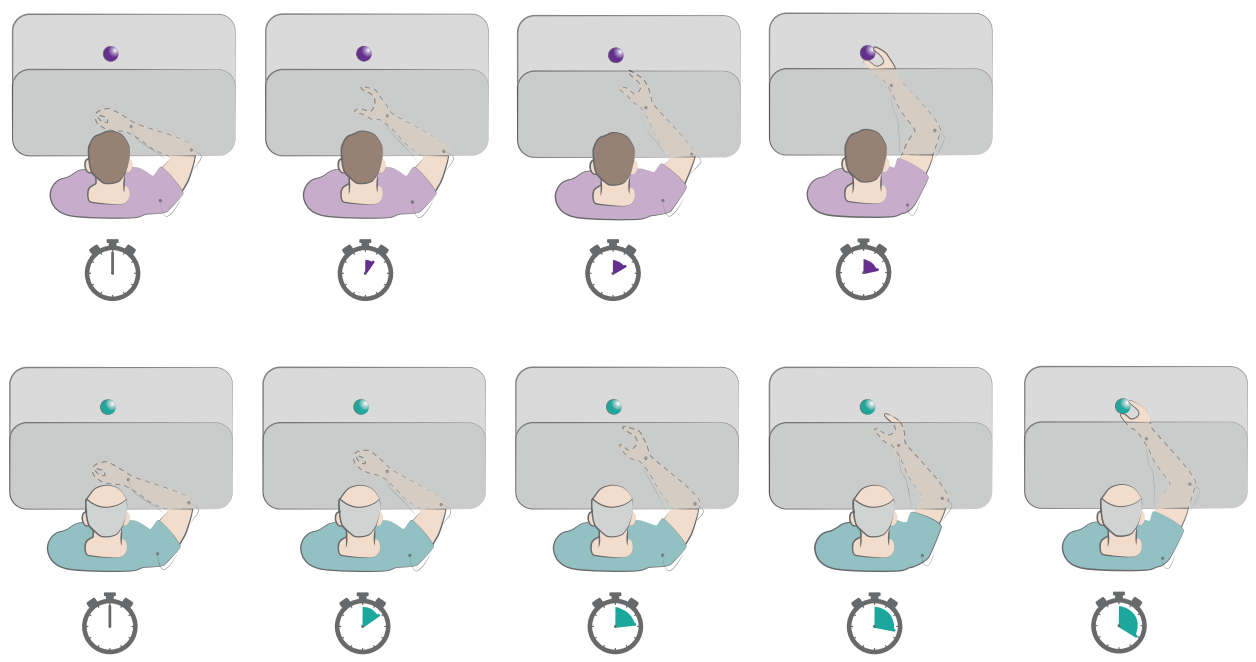

Fig. 4. Reach-to-grasp task. In this task the subject is instructed to reach and grasp a visible object on the table as fast as possible. The vision of the moving hand can be occluded. The depicted chronometers indicate the timing of performance by a healthy subject (violet) and a PD patient (green). The patients generally show bradykinesia of transport, as well as delayed and smaller hand aperture. Refer to text for details.

directions. The disease and its progression had a significant slowing effect on both reaching (decreased arm peak velocity and acceleration) and grasping (decreased peak velocity of finger opening, increased percentage of grasp time spent to achieve maximal finger aperture), particularly in the initial phase of both components. This suggested that there is not a major impact of muscle weakness, i.e., control of the necessary strength of proximal and/or distal effectors, which would have equally interested all the movement phases. In the advanced stage group, L-dopa administration led to significant improvements in the kinematics of reaching, while only the peak velocity of finger opening, hence the initial phase of grasping, was significantly improved. Therefore, dopaminergic medication seemed not to have an important effect on the BG circuits involved in the control of the grasp components, which are also less represented in BG than the reach components [192]. In PD, cortical circuitry may take over the grasp control and the coordination of grasping with reaching may then fail because of a delay in signal propagation from BG to cortical circuitry, a communication needed to temporally coordinate the finger opening with the transport movement, and which cannot be totally restored with L-dopa medication (even though Castiello et al. [44] had reported a positive medication effect on the correlations between parameters measured from the transport and those from manipulation components). These coordination dysfunctions might also be related to abnormal beta oscillations:
Vissani et al. [193] showed that amplitude and duration modulations of beta bursts are informative of the reach-to-grasp task phases, and that a lack of such informative modulation reflects pathological kinematics. Interestingly, in this study, an asymmetric inter-hemispheric striatal dopamine loss was associated with a decrease of beta bursts information in coding the movement phases and worse inter-joint movement coordination.

In experiments of Castiello et al. [194] a simultaneous perturbation of both size and location of an object to grasp determined a not synchronous, but sequential response of reach and grasp components by PD subjects. However, Rand et al. [195] revealed that PD patients were able to use the same control law as healthy subjects for governing the distance from the target of the hand aperture closure, generally shorter in the PD population $[187,188]$. This similarity was confirmed despite a perturbation of target location, suggesting that the observed shorter aperture closure distance may be substantially due to bradykinesia of transport and hypometria of grip aperture [195]. In the work of Negrotti et al. [190] the finger opening parameter was more sensitive to the object size for controls than for PD patients. This could have been due to an impairment in transforming visual target information into grasp opening, or simply in implementing initial grasp parameters for larger objects. The off-state advanced group showed instead a greater effect of object size on reach duration as compared with the other groups. This effect also can 
be explained considering longer times to grasp larger objects, which might have influenced the reach component because of the temporal coordination between the two movement phases. Overall, the intrinsic and extrinsic object properties induced similar effects on the initial kinematics of reaching and grasping within all the experimental groups, as also shown in earlier works [196-198]. Therefore, Negrotti and colleagues proposed that PD reach-to-grasp disorders do not lie in the wrong transformation of perceived object features into programmed movement parameters, but rather in the actual implementation of these parameters. Then, the decreasing of the relative duration of the deceleration with respect to the acceleration reaching phase that was observed in the PD groups was due to a lengthening of the acceleration, and did not vary with disease progression. This is interpretable again as impairment in the effective implementation of movement parameters. In fact, the final phase of the movement lengthened, in absolute sense, with disease progression, thus also the online movement control might be affected in PD advanced stages. A final suggestion by authors was that BG may serve to store movement parameters as kinematic events in a buffer, progressively provided by the SMA, which plans and temporally links them (phases of the reach and of the grasp components), and implemented in movement execution via connection from BG to the motor cortex [190].

Moreover, patients seem unable to appropriately time the deployment of the prehension components and specify the correct hand shape not only with respect to the structural features of the object but also with respect to the action goals. In [199], Ansuini and colleagues investigated how the need to perform a second action ensuing the grasping of a bottle, i.e., placing it on a specific spot or pouring the water within a container, may affect the multi-digits prehensile movement in PD patients. Control participants exhibited a shorter movement duration for the "pour" and the "place" conditions than for the "grasp" one, while for patients the movement times were similar across task conditions. For both controls and patients' groups the hand preshaping changed when the task required a second step of the motor sequence (pour and place vs. grasp), but only for controls the modulation of hand kinematics depended on the goal of the action (pour vs. place). Furthermore, in healthy subjects the necessity of a second action influenced the pattern of fingers' extension since the initial phase of the movement. Instead, for PD patients such differential pattern appeared only at late phases. This delay in the adaptation of shaping of the hand is coherent with previous literature reporting delayed onset of the PD patients' grasping with respect to the onset of the reaching [200]. They seem to hold off from executing the grasping until visual feedback of their hand is available, in order to correctly modulate the hand shape to the contours of the specific object to grasp [188]. In the work of Ansuini et al. [199] this impairment was confined to more distal joints, i.e., the joints concerned more with the final grasping. This hints at a difficulty in establishing contact points, coherently with the results of Bertram and colleagues [201], who found an increased variability of the endpoint as the hand approaches the object in the PD group with respect to controls, interpreted as impaired ability to optimally coordinate synergistic movements.

The fact that PD patients did not modulate their movement pattern and duration with the subsequent action to perform can be also explained with an involvement of BG in advance planning of movements, or in the process of forward modelling. In the neural network model of Molina-Vilaplana et al. [202], motor signals from specific cortical channels, at which the movement is planned and generated, are modulated by the gating of BG-thalamocortical loops and by reafferent proprioceptive signals related to the specific phase of the reaching. In this view, dysfunctional BG circuits may affect the ability to correctly time and modulate hand shaping in the specific task, due to a bad management of the different forward internal models related to the required prehension components [199], in line with the results and suggestions of Negrotti et al. [190]. Furthermore, in a series of studies, Straulino and colleagues [203-205] employed some variations of the reach-to-grasp paradigm in which subjects were asked to interact, communicate or compete through the graspable objects. Results from these studies demonstrated that the kinematic pattern of the reach-to-grasp movement is altered in the social condition for both healthy and PD on-medication patients. In particular, with respect to the "individual" movement, the "social" movement was characterized by longer initiation times, longer movement durations and deceleration times for both reaching and grasping phases, lower peak velocities, anticipated maximum grip aperture time. Instead, for PD off-medication patients there was no significant effect of experimental conditions on the reach-to-grasp kinematics. This suggests a role of the dopamine acting on corticostriatal synapses in the mechanisms of translation of social intentions into motor behaviors. 


\section{BEYOND OBSERVATIONS: STIMULATION STUDIES AND COMPUTATIONAL MODELS}

All the results discussed above involve the observation of the kinematics, often associated with the recording of neural activity, during the performance of reach and grasp tasks. However, it is difficult to find causal relationships and achieve an understanding of the functional network subserving the movements without perturbations. Supplement findings on reaching and grasping in PD have indeed come from invasive and non-invasive stimulation studies. These works exploit the perturbation of local or broad neural activity that manifests as changes in the behavior, allowing researchers to test or formulate new hypotheses on the neural substrates of the observed abnormal functioning, as well as to conceive new treatments.

Among the existing techniques, the low frequency repetitive transcranial magnetic stimulation (rTMS) over the motor cortex had contradictory effects on movement performances in PD patients. Quantitative improvements in the motor subscale of the UPDRS have been reported [206, 207], as well as in simple pointing movements, but not in reach-to-grasp movements [208]. Instead, the preliminary study of Thanakamchokchai and colleagues [209] suggested that high frequency rTMS to M1 may improve reachto-grasp execution in PD patients, whereas rTMS to dorsolateral prefrontal cortex may improve visuospatial processing. In [210], Palomar et al. studied reaching movements in a simple choice RT task applying a paired-pulse TMS over the right posterior parietal cortex and the right primary motor area. Healthy controls showed a significant facilitation of motor evoked potential amplitudes with a certain combination of interstimulus interval and conditioning stimulus. Patients did not show this functional interaction, but those with less impaired parietomotor interaction were faster in executing reaching movements. This implies that bradykinesia may be at least partially due to a reduced efficacy of the parieto-motor functional connectivity.

The use of transcranial direct current stimulation (tDCS) in PD is relatively recent (for a review, see [211]). It may improve the UPDRS III and the speed and force of upper limb movements, when applied to primary motor cortex [212], or to SMA [213].

As mentioned above, STN stimulation through DBS improves mainly the speed, amplitude and less the rhythm of upper limb movements [32,214], while it may fail to improve visuomotor impairments [215]. In a study by Pötter-Nerger et al. [216] PD patients with STN-DBS were instructed to perform several externally cued hand movements. Kinematic data revealed that STN-DBS improved time and maximal velocity of the hand transport within the combined reach-to-grasp movement and the isolated pointing movement (even if they remained inferior to controls), without affecting the delay of grip aperture and closure in the grasp movement. This differential impact of DBS on the bradykinesia of the proximal transport phase as compared to the distal grasp phase is in line with the results reported above concerning the differential involvement of the reach-to-grasp components. However, it is in contrast to the results of Dafotakis et al. [217], who found that bilateral stimulation of the STN ameliorates bradykinesia for both distal and proximal arm muscles in a reachto-grasp task without external cues, even though the grasp component was differentially improved. In Tamés et al. [214] bilateral and contralateral STNDBS affected similarly the proximal and distal hand movements, but only bilateral stimulation resulted optimal for proximal arm movements.

The STN-DBS can also improve the kinematics of sequential arm movements at the expense of accuracy [218], thus influencing the speed-accuracy trade-off. In a work of David and colleagues [219], PD patients with bilaterally implanted STN-DBS device performed a memory-guided sequential reaching task. The bilateral DBS significantly increased both finger and eye velocity, while it increased the finger endpoint errors. Neumann and colleagues [220] asked healthy controls and PD patients, in both the state "on" and state "off" of STN-DBS, to reach for eccentric targets appearing on a display. Independently of the clinical motor sign improvement, the DBS led to a significant decrease in reaction and movement times in an inverted with respect to the normal hand - display cursor mapping condition, while increasing the deviations from the straight path in the trajectories of patients. STN-DBS hence reduced the adaptation of RT and movement velocity to the cognitive demands of the task, causing faster but more erroneous movements, in accordance with the results of David et al. [219]. Therefore, even if BG has been depicted as an actor in both the control of movement gain [128, 221] and in coordination [134, 189], the STN-DBS may work on BG-cortical circuits to facilitate the control of intensive aspects of movement, such as velocity, but impairing those related to control of coordinative aspects, such as spatial accuracy. These effects seem 
to be similar to the effects of dopamine replacement therapies [134, 222]. However, some improvements in coordination with DBS had been reported previously [223-225]. Schettino and colleagues [225] showed an improvement in the coordination for hand preshaping, specifically ameliorating the coupling of hand aperture and finger abduction during grasping, even when vision of the moving hand was prevented. DBS seems indeed to normalize the activity patterns in the SMA and premotor cortex [226] and improve proprioception [227], key features for effective arm motion coordination.

The mechanisms through which DBS ameliorates bradykinetic symptoms are still under discussion [228-230]. Increasing subthalamic stimulation reduces beta power and increases gamma power in ipsilateral cortex [32], frequency bands that are related in some measure to bradykinetic signs, as mentioned above. Through the fiber tracking technique, Neumann and colleagues [220] observed that the modulation of RT with DBS in PD patients correlated with the amount of fibers in the hyperdirect pathway that were stimulated by active electrodes. A computational firing rate model of the cortex-BGthalamic circuit was then designed to simulate the BG activity of patients. In this model, the balance between the BG direct ("go" function) and indirect ("no-go" function) pathways determined how fast the movement was executed, while the hyperdirect pathway prevented execution of incorrect prepotent responses in case of conflicts. The DBS effects on indirect and hyperdirect pathways were modelled as the patients' UPDRS improvement (between "off" and "on") and the stimulated fiber count, respectively. To evaluate the individual contribution of these pathways to the "on" state performances, an alteration of the hyperdirect pathway was simulated as a disruption of monosynaptic cortico-STN signaling, whereas an alteration of the indirect pathway was simulated as a disruption of cortical input to striatal D2 neurons. The alteration of the hyperdirect pathway best explained the results in reaction times in DBS-on patients, in line with the fiber tracking results, while the alteration of the indirect pathway best explained the movement times data of those patients. This led authors to suggest that DBS modulates both cognitive aspects of motor preparation through the hyperdirect pathway and movement kinematics through the indirect pathway [220]. The computational firing rate model of Moroney et al. [231] aimed at replicating elbow flexion and extension movements of PD patients and demonstrated that plausible explanations of the ben- eficial effects of DBS in improving the symptoms of bradykinesia are: a stimulation-induced direct inhibition of STN cell bodies, a partial synaptic failure of the STN efferent projections to target nuclei, or excitation of inhibitory afferent axons projecting to the STN. However, the mechanisms of action of DBS may lie in a change of the firing pattern rather than in the firing rate, as suggested by the network model of Rubin and Terman [232], or even in a more complex set of phenomena described in the work of Benabid and colleagues [233].

This example of integration between clinical and computational studies and those presented in earlier sections show how general modelling of normal and lesioned BG has been fundamental to test neuroscientific hypotheses on PD. It is however critical to discriminate between functional models, describing each area according to its role and its interactions with the other areas in obtaining the final behavior, and pure neural models, describing each area as a network of neurons and deriving the functions from the neural interactions. Regarding the first category of models, each of those proposed so far focused on simulating the BG functions in a specific context, while disregarding others (for a review, see [234]). For instance, according to the "action selection" theory, BG have evolved to process distinct ongoing cortical streams signaling competing possible actions, and to appropriately select one of those while suppressing the others on the basis of the context, experience and ultimate goals [235]. This approach has proved successful in discrete selection situations such as mice in survival or foraging tasks [236] and in explaining the progressive reliance on the goal-directed mode of action control in parkinsonian patients [72]. Shortcomings of this approach appear, however, when the focus is on reproducing the impact of PD on motor behavior in the particular way the patient moves, not what action he or she chooses to perform [9]. Complications occur especially when modelling motivated modulation of a continuous variable such as velocity in motor tasks [237]. While a line of research focused on the role of ventral striatum in rewardseeking (i.e., explicitly motivated) behavior [238] and tonic dopamine's role in the control of response vigor by applying reinforcement learning theory [55], recently Dudman and colleagues $[54,56]$ attempted to outline a functional scheme of the dorsal BG circuit that could account for the (implicitly motivated) modulation of movement kinematics, as observed in the experiments of Mazzoni et al. [26]. In their view, $\mathrm{BG}$ massively integrate cortical commands to directly 
produce motor vigor signals. Bradykinetic and akinetic behaviors simply emerge as consequences of an unbalance in the average strength of the direct and indirect corticostriatal synaptic connections.

Regarding the second category, the works of Cutsuridis and colleagues [239, 240] pointed toward a comprehensive network model for studying how patterns of dopamine depletion in the BG-corticospinal circuit contribute to PD bradykinesia. The main hypotheses were the following. The dopaminergic dysfunction in the SNc disrupts the pattern of movement-related responses in the primary motor and parietal cortices, which results in a loss of directional specificity of reciprocal and bidirectional cells in the motor cortex. These changes prevent a sufficiently fast recruitment of the appropriate level of muscle force (coherent with the hypothesis by Berardelli et al. [21] of inappropriate dynamics scaling to the movement parameters), causing an increase in mean reaction time and velocity. In their model, the BG act as a gate for the cortical commands, through a voluntary, scalable "go" signal that multiplies an arm movement difference vector, computed in the parietal cortex from a comparison of a target position vector with the current perceived position vector. The product excites the primary motor cortex, thus volitional-sensitive velocity and non-specific cocontractive commands are generated and activate the lower spinal centers. Several, distinct dopaminergic signals modulate the activity of these cells, both in the parietal and the primary motor cortices. Extension-related and flexion-related motor cells are differentiated and modulated by different dopamine signals, which let the authors flexibly model the case of parkinsonian depletion. Importantly, dopamine also modulates the activity of key spinal centers [190]. The network was able to reproduce most of the bradykinetic signatures of PD patients, as well as the anomalies in cells and muscles' activation dynamics. Furthermore, the model envisioned a functional role of bidirectional primary motor cells for the coactivation of antagonist muscles under the influence of the voluntarily scalable "go" signal from the BG. It also predicted that the repetitive triphasic pattern of muscle activity observed in PD arises from the oscillatory activity of the BG output structures. However, it did not give a detailed representation of these nuclei. As mentioned above, Molina-Vilaplana and colleagues [202] used a more detailed BG-cortical loop model, starting from the work of ContrerasVidal et al. [241], to explore the possibility that the alterations of the kinematic pattern in prehensile movements of PD patients primarily arise from an altered neuronal activity located in the networks of cholinergic interneurons in the striatum. Each modelled interneuron receives a cortical input that is correlated with the cortical input to the striatal projection neuron associated with the same functional loop. Interneurons then interact with each other via lateral inhibitory connections and their activities are modulated by dopamine levels: the influence of cortical inputs on these activities decreases monotonically with dopamine depletions. Two segregated cortical visuomotor channels are simulated: one related to grip formation, another one related to hand transport. BG consist of two neural modules, each of them involved in motor modulation of one visuomotor channel, through time-varying pallido-thalamic gating signals. The hand preshaping dynamics during prehension emerges from the combined action of the feedforward cortical motor command, modulated by $\mathrm{BG}$, and the temporal coordinative role of proprioceptive reafferent information related to the transport phase of the movement: a specialized cell in the model detects the maximum in transport deceleration, which triggers target acquisition neurons in the grasp channel to sequentially switch the desired subprogram from maximum grip aperture to the aperture corresponding to the object size. Simulations revealed, under PD conditions, a restricted dynamical range of signal values at the thalamus and the lack of synchronization in the gating signals related to the - instead synchronous - cortical plans. Synchronicity was also lost between manipulation and transport components, with a delay of the onset of grasping especially for smaller simulated objects, as experimentally observed in PD patients [200]. The model indeed predicted a deficit, subsequent to dopamine depletion, for interneurons in temporally coordinate striatal outputs in order to execute parallel motor programs required for hand prehension.

\section{CONCLUSIONS}

Reaching and grasping are essential movements for our daily life and are performed by healthy subjects with high accuracy, comfort speed and minimal effort. We have seen how PD leads instead to a variety of dysfunctions associated with this task, highlighting both the inherent complexity of the movement and the multifaceted nature of this pathology. Observation of PD patients' behaviors in upper limb motor tasks has helped outline a general motor control pro- 
file of the disease, yielding theories on the plausible mechanisms of dysfunction.

As for movement programming, a first role of the BG in this phase has been proposed for the amplitude planning, given the results on reaching extent errors and variability and the related EMG insufficient activity. Hypokinetic movements may result from inappropriate BG modulation of cortical signals as well as inappropriate tuning of spinal interneuron activity, which determine an inappropriate scaling of the motor commands to the task requirements. Second, the ability to use predictive models to program and control movements may be affected too. PD patients seem indeed to be impaired in an internallybased feedforward mode (involving medial circuits) of controlling voluntary movements, thus exaggeratedly rely on an externally-guided feedback mode (involving lateral circuits), that is a compensation through sensory guidance yielding improvements in performance $[8,242]$. Confirmations of this mechanism came also from neurophysiological studies on the readiness brain potential. Non-visually guided tasks have demonstrated a fundamental and progressive kinesthetic deficiency caused by a distorted central processing of proprioceptive information, justifying patients' visual-based closed-loop strategies. This promotes the theory of BG (and SMA) contextual involvement in online motor control, particularly in the fine control of movements based on proprioception.

Cortical and muscular activation anomalies seem to favor a motor range limitation and/or a motor output extreme variability, exerting a detrimental effect both on preparing actions (delayed initiation, or akinesia) and on carrying them out (slowed execution, or bradykinesia). In movement preparation, dopamine deficiency appears to cause excessive synchronization and inter-regional coherence at beta band frequencies of motor related areas, which precludes correct movement releasing. The subthalamic nucleus' oscillatory synchronization plays instead a major role in the inhibitory control. Not only patients may prolong their initiation time to reduce risk of errors, but their capability of modifying motor plans during the reaction time is also limited, forcing them to defer these adjustments to the execution time, which fosters bradykinesia.

Both the peak kinematics and the temporal structure of the reach-to-grasp are impaired in PD. In particular, both reaching and grasping are affected by the disease and its progression, but a greater impact is seen on initial phases of both components. The differential effects of levodopa medication on reaching with respect to grasping informed on a limited influence of dopamine-related circuits specifically on the second component. The DBS can instead improve both speed and coordination between phases, through mechanisms of normalization in the temporal dynamics of cortico-BG activity that are still debated. Nonetheless, a fundamental role for BG has been hypothesized in integrating cortical information to synchronize the transport with the prehension for hand preshaping, optimally with respect to object features and task goals.

Overall, coordinative along with intensive troubling aspects of PD patients' upper limb movements shed lights on a global, and progressive, maladaptation to an altered sensorimotor apparatus. Interestingly, most of the kinematic issues in movement execution appear as arising from unintentional - and imperfect - compensations to ensure the final result of reaching the object in presence of a tradeoff between accuracy and vigor. On the other hand, task requirements and its goals, as well as individual, implicit states impose an only recently recognized influence on movement behavior. PD patients' bradykinesia in self-paced tasks and the beneficial effects of sensory cues and urgency corroborate the interpretation of inability to internally maximize their movement kinematics. Tasks requiring low level of accuracy have shown that the scarce vigor of movements may result primarily from central deficits in the motor production associated with an individual motor motivation. This motivation suffers from the PD patients' excessive sensitivity to the energetic cost of the movement, signaled by low levels of tonic nigrostriatal dopamine. Dopaminergic drugs as well as DBS treatment help shift the aberrant speed-accuracy balance towards the restoring of motor vigor. A motivation signal may also assist the translation of social intentions into optimal motor behaviors, given that patients off medication seem to be more indifferent to action goals. This aspect, however, needs further experimental confirmations.

\section{Future perspectives}

The simultaneous presence of issues that directly stem from cortico-BG malfunctions and issues indirectly stemming from attempts to compensate these malfunctions makes for a complex picture of reach and grasp in PD. This led to the development of progressively more elegant experimental designs trying to disentangle the different components. Future 
behavioral studies should address the still open questions, such as those concerning the origins of the distortion in the speed selection. This particular issue might be tackled by struggling to reduce betweenand within-group differences related to the management of accuracy constraints, for instance defining individual indices of task difficulty along the dimensions of effort and accuracy on the basis of each subject's performance levels, in the wake of the work by Gepshtein and colleagues [60]. Other challenges will be trying to separate more clearly planning and preparation from execution problems, and to elucidate the role of the BG in forward modelling and in online feedback control. This will also help resolve the controversies around the relative proportion of deceleration and acceleration in the movement profiles of patients for the same task. In the attempt to compare results with previous literature, the disease stage, inter-individual variability as well as any confound of attentional interferences and potential cognitive impairments should be taken into account.

A key element for future investigations in the field will be to complement observational studies with perturbation studies involving cortical stimulations. Recording studies have already allowed researchers to associate behavioral dysfunctions with anomalies of brain activity. For instance, EEG analysis has been helpful in identifying predictors of bradykinetic signs (and their improvement), as activity variations mainly in the delta and gamma bands have been associated with distinct parameters of movement execution. In addition to this kind of studies, correlations may be established by direct (invasive or not) intervention on the interested circuits, as stimulation experiments have shown. Stimulation studies might have an even more relevant role in the future, thanks to the possibility of exerting safe and reversible perturbations while recording neural activity. This recently allowed Muthuraman and colleagues [32] to relate DBS-induced changes in speed, amplitude and rhythm of patients' hand movements to the evolution of motor, premotor and STN activities individually, by analyzing the subnetworks' preferred frequency of oscillation with the EEG. Furthermore, the recent technique of adaptive DBS [230], i.e., stimulation automatically adjusted and tailored to the patient's clinical state, may provide also further insights into the movement pathophysiology. The stimulation can be triggered by non-neural, movement biomarkers, for example computed by processing signals coming from wearable sensors monitoring the upper limb joints' configuration. By comparing on- and off-stimulation states, more reliable associations between the local circuitry and affected spatio-temporal parameters would be obtained. Importantly, these biomarkers evolve with the course of the disease and can powerfully discriminate among different motor phenotypes of $\mathrm{PD}$, facilitating the tailoring of the stimulation protocol to the specific patient.

Once pathological behaviors have been mapped into some neural substrates, novel hypotheses on dysfunctional mechanisms can be tested through computational modelling studies. In face of the obvious limitations in biological realism, virtual simulations provide extreme flexibility, since pathological states can be simulated at different levels of the system, locally or globally, and different assumptions can be first translated into both circuital and functional model features and then verified in the emerging behavior of the simulated network. On one side, purely functional models will be useful for efficiently embedding a BG unit in a braininspired architecture and simulating motor control and learning mechanisms in an artificial agent [243, 244], as well as reproducing overall characteristics of the disease in silico. On the other side, neural networks modelling [245, 246] can capture microstructural features which sound essential for studying the dynamical properties of the altered circuits. Spiking neural networks, in particular, can prove whether kinematic anomalies arise from alterations in the dynamics and activity patterns of specific BG nuclei or pathways, eventually localizing them. Perturbations can be modelled as well: in this context, the work of Neumann and colleagues [220] is a meaningful example of how the integration of behavioral test, stimulation experiment and computational validation can bring about new useful insights both for neuroscientific research and for DBS treatment. We envisage that this integration may be the right key to solve the open debates on the reaching and grasping anomalies of parkinsonian patients, leading to new knowledge and more complete understanding of the disease and of the human motor control in general. Moreover, it might acquire clinical relevance in the future since computational modelling will enable to find a set of individual motor parameters that are fundamental for outlining a motor control profile of the PD patient. Together with the refinement of motor assessment techniques necessary for clinical investigations of more complex aspects of movements, this effort will finally provide quantitative tools to establish predictors of disease trajectories as well 
as reliably monitor the effects of both invasive and non-invasive therapies.

\section{ACKNOWLEDGMENTS}

$\mathrm{AF}$ and $\mathrm{EF}$ have received funding from the European Union's Horizon 2020 Framework Programme for Research and Innovation under the Specific Grant Agreements No. 785907 (Human Brain Project SGA2) and No. 945539 (Human Brain Project SGA3). AM was partially funded by the Clinical Research Grant of Fondazione Marlene and Paolo Fresco Institute for Parkinson's and Movement Disorders "Determining the neural correlates of targeted non-invasive neuromodulation and speech therapy for a personalized treatment of speech symptoms in Parkinson's disease". Authors thank Irene Mannari for her help with illustrations.

\section{CONFLICT OF INTEREST}

The authors have no conflict of interest to report.

\section{REFERENCES}

[1] Hallett M, Shahani BT, Young RR (1977) Analysis of stereotyped voluntary movements at the elbow in patients with Parkinson's disease. J Neurol Neurosurg Psychiatry 40, 1129-1135.

[2] Dick JP, Rothwell JC, Day BL, Cantello R, Buruma O, Gioux M, Benecke R, Berardelli A, Thompson PD, Marsden CD (1989) The Bereitschaftspotential is abnormal in Parkinson's disease. Brain 112 (Pt 1), 233-244.

[3] Schneider JS, Diamond SG, Markham CH (1987) Parkinson's disease: Sensory and motor problems in arms and hands. Neurology 37, 951-951.

[4] Giladi N, Treves TA, Simon ES, Shabtai H, Orlov Y, Kandinov B, Paleacu D, Korczyn AD (2001) Freezing of gait in patients with advanced Parkinson's disease. $J$ Neural Transm 108, 53-61.

[5] Debû B, De Oliveira Godeiro C, Lino JC, Moro E (2018) Managing gait, balance, and posture in Parkinson's disease. Curr Neurol Neurosci Rep 18, 23.

[6] Goetz CG, Tilley BC, Shaftman SR, Stebbins GT, Fahn S, Martinez-Martin P, Poewe W, Sampaio C, Stern MB, Dodel R, Dubois B, Holloway R, Jankovic J, Kulisevsky J, Lang AE, Lees A, Leurgans S, LeWitt PA, Nyenhuis D, Olanow CW, Rascol O, Schrag A, Teresi JA, van Hilten JJ, LaPelle N, Movement Disorder Society UPDRS Revision Task Force (2008) Movement Disorder Society-sponsored revision of the Unified Parkinson's Disease Rating Scale (MDS-UPDRS): Scale presentation and clinimetric testing results. Mov Disord 23, 2129-2170.

[7] Flowers K (1975) Ballistic and corrective movements on an aiming task. Intention tremor and parkinsonian movement disorders compared. Neurology 25, 413-421.
[8] Flowers K (1976) Visual "closed-loop" and "open-loop" characteristics of voluntary movement in patients with Parkinsonism and intention tremor. Brain 99, 269-310.

[9] Mazzoni P, Shabbott B, Cortés JC (2012) Motor control abnormalities in Parkinson's disease. Cold Spring Harb Perspect Med 2, a009282.

[10] Parma V, Zanatto D, Straulino E, Scaravilli T, Castiello U (2014) Kinematics of the reach-to-grasp movement in vascular parkinsonism: A comparison with idiopathic Parkinson's disease patients. Front Neurol 5, 75.

[11] DeLong MR (1990) Primate models of movement disorders of basal ganglia origin. Trends Neurosci 13, 281-285.

[12] DeLong M, Wichmann T (2010) Changing views of basal ganglia circuits and circuit disorders. Clin EEG Neurosci 41, 61-67.

[13] Mink JW (1996) The basal ganglia: Focused selection and inhibition of competing motor programs. Prog Neurobiol 50, 381-425.

[14] Bar-Gad I, Bergman H (2001) Stepping out of the box: Information processing in the neural networks of the basal ganglia. Curr Opin Neurobiol 11, 689-695.

[15] Bevan MD, Magill PJ, Terman D, Bolam JP, Wilson CJ (2002) Move to the rhythm: Oscillations in the subthalamic nucleus-external globus pallidus network. Trends Neurosci 25, 525-531.

[16] Woodworth RS (1899) Accuracy of voluntary movement. Psychol Rev Monograph Suppl 3, i-114.

[17] Johns RJ, Draper IT (1964) The control of movement in normal subjects. Bull Johns Hopkins Hosp 115, 447-464.

[18] Draper IT, Johns RJ (1964) The disordered movement in parkinsonism and the effect of drug treatment. Bull Johns Hopkins Hosp 115, 465-480.

[19] Hallett M, Khoshbin S (1980) A physiological mechanism of bradykinesia. Brain 103, 301-314.

[20] Hallett M, Marsden CD (1979) Ballistic flexion movements of the human thumb. J Physiol 294, 33-50.

[21] Berardelli A, Dick JP, Rothwell JC, Day BL, Marsden CD (1986) Scaling of the size of the first agonist EMG burst during rapid wrist movements in patients with Parkinson's disease. J Neurol Neurosurg Psychiatry 49, 1273-1279.

[22] Berardelli A, Accornero N, Argenta M, Meco G, Manfredi M (1986) Fast complex arm movements in Parkinson's disease. J Neurol Neurosurg Psychiatry 49, 1146-1149.

[23] Berardelli A, Hallett M, Rothwell JC, Agostino R, Manfredi M, Thompson PD, Marsden CD (1996) Single-joint rapid arm movements in normal subjects and in patients with motor disorders. Brain 119, 661-674.

[24] Berardelli A, Rothwell JC, Thompson PD, Hallett M (2001) Pathophysiology of bradykinesia in Parkinson's disease. Brain 124, 2131-2146.

[25] Klockgether T, Dichgans J (1994) Visual control of arm movement in Parkinson's disease. Mov Disord 9, 48-56.

[26] Mazzoni P, Hristova A, Krakauer JW (2007) Why don't we move faster? Parkinson's disease, movement vigor, and implicit motivation. J Neurosci 27, 7105-7116.

[27] Baraduc P, Thobois S, Gan J, Broussolle E, Desmurget M (2013) A common optimization principle for motor execution in healthy subjects and parkinsonian patients. J Neurosci 33, 665-677.

[28] Postuma RB, Berg D, Stern M, Poewe W, Olanow CW, Oertel W, Obeso J, Marek K, Litvan I, Lang AE, Halliday G, Goetz CG, Gasser T, Dubois B, Chan P, Bloem BR, Adler CH, Deuschl G (2015) MDS clinical diagnostic criteria for Parkinson's disease. Mov Disord 30, 1591-1601. 
[29] Kishore A, Espay AJ, Marras C, Al-Khairalla T, Arenovich T, Asante A, Miyasaki J, Lang AE (2007) Unilateral versus bilateral tasks in early asymmetric Parkinson's disease: Differential effects on bradykinesia. Mov Disord 22, 328-333.

[30] Espay AJ, Giuffrida JP, Chen R, Payne M, Mazzella F, Dunn E, Vaughan JE, Duker AP, Sahay A, Kim SJ, Revilla FJ, Heldman DA (2011) Differential response of speed, amplitude, and rhythm to dopaminergic medications in Parkinson's disease. Mov Disord 26, 2504-2508.

[31] Heldman DA, Giuffrida JP, Chen R, Payne M, Mazzella F, Duker AP, Sahay A, Kim SJ, Revilla FJ, Espay AJ (2011) The modified bradykinesia rating scale for Parkinson's disease: Reliability and comparison with kinematic measures. Mov Disord 26, 1859-1863.

[32] Muthuraman M, Palotai M, Jévor-Duray B, Kelemen A, Koirala N, Halész L, Erőss L, Fekete G, Bognér L, Deuschl G, Tamés G (2021) Frequency-specific network activity predicts bradykinesia severity in Parkinson's disease. Neuroimage Clin 32, 102857

[33] Teasdale N, Phillips J, Stelmach GE (1990) Temporal movement control in patients with Parkinson's disease. J Neurol Neurosurg Psychiatry 53, 862-868.

[34] Gordon AM (1998) Task-dependent deficits during object release in Parkinson's disease. Exp Neurol 153, 287-298.

[35] Broderick MP, Van Gemmert AWA, Shill HA, Stelmach GE (2009) Hypometria and bradykinesia during drawing movements in individuals with Parkinson's disease. Exp Brain Res 197, 223-233.

[36] Sheridan MR, Flowers KA, Hurrell J (1987) Programming and execution of movement in parkinson's disease. Brain 110, 1247-1271.

[37] Sheridan MR, Flowers KA (1990) Movement variability and bradykinesia in Parkinson's disease. Brain 113 (Pt 4), 1149-1161.

[38] Montgomery EB, Nuessen J (1990) The movement speed/accuracy operator in Parkinson's disease. Neurology 40, 269-272.

[39] Sanes JN (1985) Information processing deficits in Parkinson's disease during movement. Neuropsychologia 23, 381-392.

[40] Doan J, Whishaw IQ, Pellis SM, Suchowersky O, Brown LA (2006) Motor deficits in Parkinsonian reaching: Dopasensitivity influenced by real-world task constraint. J Mot Behav 38, 45-59.

[41] Canning CG (2005) The effect of directing attention during walking under dual-task conditions in Parkinson's disease. Parkinsonism Relat Disord 11, 95-99.

[42] Camarda R, Camarda C, Grimaldi S, Camarda LKC, Monastero R, Gangitano M (2005) Effects of levodopa oral bolus on the kinematics of the pointing movements in Parkinson's disease patients. J Neurol 252, 1074-1081.

[43] Flash T, Inzelberg R, Schechtman E, Korczyn AD (1992) Kinematic analysis of upper limb trajectories in Parkinson's disease. Exp Neurol 118, 215-226.

[44] Castiello U, Bennett KM, Bonfiglioli C, Peppard RF (2000) The reach-to-grasp movement in Parkinson's disease before and after dopaminergic medication. $\mathrm{Neu}$ ropsychologia 38, 46-59.

[45] Rand MK, Stelmach GE, Bloedel JR (2000) Movement accuracy constraints in Parkinson's disease patients. Neuropsychologia 38, 203-212.

[46] Phillips JG, Martin KE, Bradshaw JL, Iansek R (1994) Could bradykinesia in Parkinson's disease simply be compensation? J Neurol 241, 439-447.
[47] Corcos DM, Chen C-M, Quinn NP, McAuley J, Rothwell JC (1996) Strength in Parkinson's disease: Relationship to rate of force generation and clinical status. Ann Neurol 39, 79-88.

[48] Todorov E, Jordan MI (2002) Optimal feedback control as a theory of motor coordination. Nat Neurosci 5, 1226-1235.

[49] Shadmehr R, Krakauer JW (2008) A computational neuroanatomy for motor control. Exp Brain Res 185, 359-381.

[50] Schmidt L, d'Arc BF, Lafargue G, Galanaud D, Czernecki V, Grabli D, Schüpbach M, Hartmann A, Lévy R, Dubois B, Pessiglione M (2008) Disconnecting force from money: Effects of basal ganglia damage on incentive motivation. Brain 131, 1303-1310.

[51] Manohar SG, Chong TT-J, Apps MAJ, Batla A, Stamelou M, Jarman PR, Bhatia KP, Husain M (2015) Reward pays the cost of noise reduction in motor and cognitive control. Curr Biol 25, 1707-1716.

[52] Kojovic M, Mir P, Trender-Gerhard I, Schneider SA, Pareés I, Edwards MJ, Bhatia KP, Jahanshahi M (2014) Motivational modulation of bradykinesia in Parkinson's disease off and on dopaminergic medication. J Neurol 261, 1080-1089.

[53] Turner RS, Desmurget M (2010) Basal ganglia contributions to motor control: A vigorous tutor. Curr Opin Neurobiol 20, 704-716.

[54] Dudman JT, Krakauer JW (2016) The basal ganglia: From motor commands to the control of vigor. Curr Opin $\mathrm{Neu}$ robiol 37, 158-166.

[55] Niv Y, Daw ND, Joel D, Dayan P (2007) Tonic dopamine: Opportunity costs and the control of response vigor. Psychopharmacology 191, 507-520.

[56] Yttri EA, Dudman JT (2018) A proposed circuit computation in basal ganglia: History-dependent gain. Mov Disord 33, 704-716.

[57] Moisello C, Perfetti B, Marinelli L, Sanguineti V, Bove M, Feigin A, Di Rocco A, Eidelberg D, Ghilardi MF (2011) Basal ganglia and kinematics modulation: Insights from Parkinson's and Huntington's diseases. Parkinsonism Relat Disord 17, 642-644.

[58] Harris CM, Wolpert DM (1998) Signal-dependent noise determines motor planning. Nature 394, 780-784.

[59] Guigon E, Baraduc P, Desmurget M (2007) Computational motor control: Redundancy and invariance. J Neurophysiol 97, 331-347.

[60] Gepshtein S, Li X, Snider J, Plank M, Lee D, Poizner H (2014) Dopamine function and the efficiency of human movement. J Cogn Neurosci 26, 645-657.

[61] Glickstein M, Stein J (1991) Paradoxical movement in Parkinson's disease. Trends Neurosci 14, 480-482.

[62] Majsak MJ, Kaminski T, Gentile AM, Flanagan JR (1998) The reaching movements of patients with Parkinson's disease under self-determined maximal speed and visually cued conditions. Brain 121 (Pt 4), 755-766.

[63] Ballanger B, Thobois S, Baraduc P, Turner RS, Broussolle E, Desmurget M (2006) "Paradoxical Kinesis" is not a hallmark of Parkinson's disease but a general property of the motor system. Mov Disord 21, 1490-1495.

[64] Majsak MJ, Kaminski T, Gentile AM, Gordon AM (2008) Effects of a moving target versus a temporal constraint on reach and grasp in patients with Parkinson's disease. Exp Neurol 210, 479-488.

[65] Bieńkiewicz MMN, Young WR, Craig CM (2014) Balls to the wall: How acoustic information from a ball in motion 
guides interceptive movement in people with Parkinson's disease. Neuroscience 275, 508-518.

[66] Cassimatis C, Liu KPY, Fahey P, Bissett M (2016) The effectiveness of external sensory cues in improving functional performance in individuals with Parkinson's disease: A systematic review with meta-analysis. Int $J$ Rehabil Res 39, 211-218.

[67] Akamatsu T, Fukuyama H, Kawamata T (2008) The effects of visual, auditory, and mixed cues on choice reaction in Parkinson's disease. J Neurol Sci 269, 118-125.

[68] Praamstra P, Stegeman DF, Cools AR, Horstink MWIM (1998) Reliance on external cues for movement initiation in Parkinson's disease. Evidence from movement-related potentials. Brain 121, 167-177.

[69] Schenk T, Baur B, Steude U, Bötzel K (2003) Effects of deep brain stimulation on prehensile movements in PD patients are less pronounced when external timing cues are provided. Neuropsychologia 41, 783-794.

[70] Ginis P, Nackaerts E, Nieuwboer A, Heremans E (2018) Cueing for people with Parkinson's disease with freezing of gait: A narrative review of the state-of-the-art and novel perspectives. Ann Phys Rehabil Med 61, 407-413.

[71] Bieńkiewicz MMN, Rodger MWM, Young WR, Craig CM (2013) Time to get a move on: Overcoming bradykinetic movement in Parkinson's disease with artificial sensory guidance generated from biological motion. Behav Brain Res 253, 113-120.

[72] Redgrave P, Rodriguez M, Smith Y, Rodriguez-Oroz MC, Lehericy S, Bergman H, Agid Y, DeLong MR, Obeso JA (2010) Goal-directed and habitual control in the basal ganglia: Implications for Parkinson's disease. Nat Rev Neurosci 11, 760-772.

[73] Bek J, Gowen E, Vogt S, Crawford TJ, Poliakoff E (2021) Action observation and imitation in Parkinson's disease: The influence of biological and non-biological stimuli. Neuropsychologia 150, 107690.

[74] Castiello U, Ansuini C, Bulgheroni M, Scaravilli T, Nicoletti R (2009) Visuomotor priming effects in Parkinson's disease patients depend on the match between the observed and the executed action. Neuropsychologia 47, 835-842.

[75] Mongeon D, Blanchet P, Messier J (2009) Impact of Parkinson's disease and dopaminergic medication on proprioceptive processing. Neuroscience 158, 426-440.

[76] Jeannerod M (1984) The timing of natural prehension movements. J Mot Behav 16, 235-254.

[77] Gosselin-Kessiby N, Kalaska JF, Messier J (2009) Evidence for a proprioception-based rapid on-line error correction mechanism for hand orientation during reaching movements in blind subjects. $J$ Neurosci 29, 3485-3496.

[78] Poizner H, Fookson OI, Berkinblit MB, Hening W, Feldman G, Adamovich S (1998) Pointing to remembered targets in 3-D space in Parkinson's disease. Motor Control 2, 251-277.

[79] Myall DJ, MacAskill MR, Anderson TJ, Jones RD (2008) Submovements in visually-guided and memory-guided reaching tasks: Changes in Parkinson's disease. Annu Int Conf IEEE Eng Med Biol Soc 2008, 1761-1764.

[80] Mongeon D, Blanchet P, Bergeron S, Messier J (2015) Impact of Parkinson's disease on proprioceptively based on-line movement control. Exp Brain Res 233, 2707-2721.

[81] Maschke M, Gomez CM, Tuite PJ, Konczak J (2003) Dysfunction of the basal ganglia, but not the cerebellum, impairs kinaesthesia. Brain 126, 2312-2322.
[82] Putzki N, Stude P, Konczak J, Graf K, Diener H-C, Maschke M (2006) Kinesthesia is impaired in focal dystonia. Mov Disord 21, 754-760.

[83] Konczak J, Krawczewski K, Tuite P, Maschke M (2007) The perception of passive motion in Parkinson's disease. J Neurol 254, 655.

[84] Konczak J, Corcos DM, Horak F, Poizner H, Shapiro M, Tuite P, Volkmann J, Maschke M (2009) Proprioception and motor control in Parkinson's disease. J Mot Behav 41, 543-552.

[85] Burke D, Hagbarth KE, Wallin BG (1977) Reflex mechanisms in Parkinsonian rigidity. Scand J Rehabil Med $\mathbf{9}$, 15-23.

[86] Moore A (1989) Vibration-induced illusions of movement are normal in Parkinson's disease: Implications for the mechanism of the movement disorder. Neural Mechanisms in Disorders of Movement, Crossman AR, Sambrook MA, eds. John Libbey, London, pp. 307-311.

[87] Moore AP (1987) Impaired sensorimotor integration in parkinsonism and dyskinesia: A role for corollary discharges? J Neurol Neurosurg Psychiatry 50, 544-552.

[88] Demirci M, Grill S, McShane L, Hallett M (1997) A mismatch between kinesthetic and visual perception in Parkinson's disease. Ann Neurol 41, 781-788.

[89] Koop MM, Hill BC, Bronte-Stewart HM (2013) Perceptual errors increase with movement duration and may contribute to hypokinesia in Parkinson's disease. Neuroscience 243, 1-13.

[90] Adamovich SV, Berkinblit MB, Hening W, Sage J, Poizner $\mathrm{H}$ (2001) The interaction of visual and proprioceptive inputs in pointing to actual and remembered targets in Parkinson's disease. Neuroscience 104, 1027-1041.

[91] Ghilardi M-F, Alberoni M, Rossi M, Franceschi M, Mariani C, Fazio F (2000) Visual feedback has differential effects on reaching movements in Parkinson's and Alzheimer's disease. Brain Res 876, 112-123.

[92] Keijsers NLW, Admiraal MA, Cools AR, Bloem BR, Gielen CCAM (2005) Differential progression of proprioceptive and visual information processing deficits in Parkinson's disease. Eur J Neurosci 21, 239-248.

[93] Tagliabue M, Ferrigno G, Horak F (2009) Effects of Parkinson's disease on proprioceptive control of posture and reaching while standing. Neuroscience 158, 12061214.

[94] Ho AK, Bradshaw JL, Iansek T (2000) Volume perception in parkinsonian speech. Mov Disord 15, 1125-1131.

[95] Marsden CD (1982) The mysterious motor function of the basal ganglia: The Robert Wartenberg Lecture. Neurology 32, 514-514.

[96] Klockgether T, Borutta M, Rapp H, Spieker S, Dichgans J (1995) A defect of kinesthesia in Parkinson's disease. Mov Disord 10, 460-465.

[97] Viallet F, Trouche E, Legallet E, Apicella P, Khalil R (1991) Differential aspects of parkinsonian akinesia as revealed by limb movement studies. In The Basal Ganglia III, Bernardi G, Carpenter MB, Di Chiara G, Morelli M, Stanzione P, eds. Springer New York, Boston, MA, pp. 645-652.

[98] Lawrence AD (2000) Error correction and the basal ganglia: Similar computations for action, cognition and emotion? Trends Cogn Sci 4, 365-367.

[99] Smith MA, Brandt J, Shadmehr R (2000) Motor disorder in Huntington's disease begins as a dysfunction in error feedback control. Nature 403, 544-549. 
[100] Tunik E, Adamovich SV, Poizner H, Feldman AG (2004) Deficits in rapid adjustments of movements according to task constraints in Parkinson's disease. Mov Disord 19, 897-906

[101] Desmurget M, Gaveau V, Vindras P, Turner RS, Broussolle E, Thobois S (2004) On-line motor control in patients with Parkinson's disease. Brain 127, 1755-1773.

[102] Flash T, Henis E (1991) Arm trajectory modifications during reaching towards visual targets. J Cogn Neurosci 3, 220-230.

[103] Novak KE, Miller LE, Houk JC (2002) The use of overlapping submovements in the control of rapid hand movements. Exp Brain Res 144, 351-364.

[104] Desmurget M, Grafton S (2003) Feedback or feedforward control: End of a dichotomy. In Taking action: Cognitive neuroscience perspectives on intentional acts, The MIT Press, Cambridge, MA, US, pp. 289-338.

[105] Desmurget M, Gréa H, Grethe JS, Prablanc C, Alexander GE, Grafton ST (2001) Functional anatomy of nonvisual feedback loops during reaching: A positron emission tomography study. J Neurosci 21, 2919-2928.

[106] Jobst EE, Melnick ME, Byl NN, Dowling GA, Aminoff MJ (1997) Sensory perception in Parkinson disease. Arch Neurol 54, 450-454.

[107] Maschke M, Tuite PJ, Krawczewski K, Pickett K, Konczak J (2006) Perception of heaviness in Parkinson's disease. Mov Disord 21, 1013-1018.

[108] O'Suilleabhain P, Bullard J, Dewey R (2001) Proprioception in Parkinson's disease is acutely depressed by dopaminergic medications. J Neurol Neurosurg Psychiatry 71, 607-610.

[109] Benecke R, Rothwell JC, Dick JP, Day BL, Marsden CD (1987) Disturbance of sequential movements in patients with Parkinson's disease. Brain 110 (Pt 2), 361-379.

[110] Agostino R, Berardelli A, Formica A, Accornero N, Manfredi M (1992) Sequential arm movements in patients with Parkinson's disease, Huntington's disease and dystonia. Brain 115 (Pt 5), 1481-1495.

[111] Weiss P, Stelmach GE, Hefter H (1997) Programming of a movement sequence in Parkinson's disease. Brain 120, 91-102.

[112] Almeida QJ, Wishart LR, Lee TD (2003) Disruptive influences of a cued voluntary shift on coordinated movement in Parkinson's disease. Neuropsychologia 41, 442-452.

[113] Kang SY, Wasaka T, Shamim EA, Auh S, Ueki Y, Lopez GJ, Kida T, Jin S-H, Dang N, Hallett M (2010) Characteristics of the sequence effect in Parkinson's disease. Mov Disord 25, 2148-2155.

[114] Currá A, Berardelli A, Agostino R, Modugno N, Puorger CC, Accornero N, Manfredi M (1997) Performance of sequential arm movements with and without advance knowledge of motor pathways in Parkinson's disease. Mov Disord 12, 646-654.

[115] Wolpert DM, Flanagan JR (2001) Motor prediction. Curr Biol 11, R729-R732.

[116] Tunik E, Houk JC, Grafton ST (2009) Basal ganglia contribution to the initiation of corrective submovements. Neuroimage 47, 1757-1766.

[117] Chevalier G, Deniau JM (1990) Disinhibition as a basic process in the expression of striatal functions. Trends $\mathrm{Neu}$ rosci 13, 277-280.

[118] Fradet L, Lee G, Dounskaia N (2008) Origins of submovements during pointing movements. Acta Psychol 129, 91-100.
[119] Doeringer JA, Hogan N (1998) Intermittency in preplanned elbow movements persists in the absence of visual feedback. J Neurophysiol 80, 1787-1799.

[120] Vaillancourt DE, Larsson L, Newell KM (2003) Effects of aging on force variability, single motor unit discharge patterns, and the structure of 10,20, and $40 \mathrm{~Hz}$ EMG activity. Neurobiol Aging 24, 25-35.

[121] Dounskaia N, Fradet L, Lee G, Leis BC, Adler CH (2009) Submovements during pointing movements in Parkinson's disease. Exp Brain Res 193, 529-544.

[122] Kato M, Kimura M (1992) Effects of reversible blockade of basal ganglia on a voluntary arm movement. $J$ Neurophysiol 68, 1516-1534.

[123] Pfann KD, Buchman AS, Comella CL, Corcos DM (2001) Control of movement distance in Parkinson's disease. Mov Disord 16, 1048-1065.

[124] Robichaud JA, Pfann KD, Comella CL, Corcos DM (2002) Effect of medication on EMG patterns in individuals with Parkinson's disease. Mov Disord 17, 950-960.

[125] Merritt KE, Seergobin KN, Mendonça DA, Jenkins ME, Goodale MA, MacDonald PA (2017) Automatic online motor control is intact in Parkinson's disease with and without perceptual awareness. eNeuro 4, ENEURO.021517.2017.

[126] Sarlegna FR, Mutha PK (2015) The influence of visual target information on the online control of movements. Vis Res 110, 144-154.

[127] Wolpert DM, Ghahramani Z, Jordan MI (1995) An internal model for sensorimotor integration. Science 269, 1880-1882.

[128] Desmurget M, Grafton ST, Vindras P, Gréa H, Turner RS (2003) Basal ganglia network mediates the control of movement amplitude. Exp Brain Res 153, 197-209.

[129] Desmurget M, Grafton ST, Vindras P, Gréa H, Turner RS (2004) The basal ganglia network mediates the planning of movement amplitude. Eur J Neurosci 19, 2871-2880.

[130] Turner RS, Grafton ST, Votaw JR, Delong MR, Hoffman JM (1998) Motor subcircuits mediating the control of movement velocity: A PET study. J Neurophysiol 80, 2162-2176.

[131] Siebner HR, Limmer C, Peinemann A, Bartenstein P, Drzezga A, Conrad B (2001) Brain correlates of fast and slow handwriting in humans: A PET-performance correlation analysis. Eur J Neurosci 14, 726-736.

[132] Grafton ST, Tunik E (2011) Human basal ganglia and the dynamic control of force during on-line corrections. J Neurosci 31, 1600-1605.

[133] Lee D, Henriques DY, Snider J, Song D, Poizner H (2013) Reaching to proprioceptively defined targets in Parkinson's disease: Effects of deep brain stimulation therapy. Neuroscience 244, 99-112.

[134] Schettino LF, Adamovich SV, Hening W, Tunik E, Sage J, Poizner H (2006) Hand preshaping in Parkinson's disease: Effects of visual feedback and medication state. Exp Brain Res 168, 186-202.

[135] Lukos JR, Snider J, Hernandez ME, Tunik E, Hillyard S, Poizner H (2013) Parkinson's disease patients show impaired corrective grasp control and eye-hand coupling when reaching to grasp virtual objects. Neuroscience 254, 205-221.

[136] Smiley-Oyen AL, Lowry KA, Kerr JP (2007) Planning and control of sequential rapid aiming in adults with Parkinson's disease. J Mot Behav 39, 103-114.

[137] Evarts EV, Teräväinen H, Calne DB (1981) Reaction time in Parkinson's disease. Brain 104, 167-186. 
[138] Jahanshahi M, Brown RG, Marsden CD (1992) Simple and choice reaction time and the use of advance information for motor preparation in Parkinson's disease. Brain 115 (Pt 2), 539-564.

[139] Kutukcu Y, Marks WJ, Goodin DS, Aminoff MJ (1999) Simple and choice reaction time in Parkinson's disease. Brain Res 815, 367-372.

[140] Bloxham CA, Mindel TA, Frith CD (1984) Initiation and execution of predictable and unpredictable movements in Parkinson's disease. Brain 107 (Pt 2), 371-384

[141] Brown RG, Jahanshahi M, Marsden CD (1993) Response choice in Parkinson's disease: The effects of uncertainty and stimulus-response compatibility. Brain 116, 869-885.

[142] Stelmach GE, Worringham CJ, Strand EA (1986) Movement preparation in Parkinson's disease. The use of advance information. Brain 109 (Pt 6), 1179-1194.

[143] Gauntlett-Gilbert J, Brown VJ (1998) Reaction time deficits and Parkinson's disease. Neurosci Biobehav Rev 22, 865-881.

[144] Johnson AM, Almeida QJ, Stough C, Thompson JC, Singarayer R, Jog MS (2004) Visual inspection time in Parkinson's disease: Deficits in early stages of cognitive processing. Neuropsychologia 42, 577-583.

[145] Carlsen AN, Almeida QJ, Franks IM (2013) Using a startling acoustic stimulus to investigate underlying mechanisms of bradykinesia in Parkinson's disease. Neuropsychologia 51, 392-399.

[146] Fernandez-Del-Olmo M, Bello O, Lopez-Alonso V, Marquez G, Sanchez JA, Morenilla L, Valls-Solé J (2013) The effects of startle and non-startle auditory stimuli on wrist flexion movement in Parkinson's disease. Neurosci Lett 548, 56-60.

[147] Haith AM, Pakpoor J, Krakauer JW (2016) Independence of movement preparation and movement initiation. $\mathrm{J} \mathrm{Neu}$ rosci 36, 3007-3015.

[148] Mirabella G, Pani P, Ferraina S (2008) Context influences on the preparation and execution of reaching movements. Cogn Neuropsychol 25, 996-1010.

[149] Manza P, Amandola M, Tatineni V, Li C-SR, Leung HC (2017) Response inhibition in Parkinson's disease: A meta-analysis of dopaminergic medication and disease duration effects. NPJ Parkinsons Dis 3, 23.

[150] Leis BC, Rand MK, Van Gemmert AWA, Longstaff MG, Lou JS, Stelmach GE (2005) Movement precues in planning and execution of aiming movements in Parkinson's disease. Exp Neurol 194, 393-409.

[151] Perfetti B, Moisello C, Lanzafame S, Varanese S, Landsness EC, Onofrj M, Di Rocco A, Tononi G, Ghilardi MF (2010) Attention modulation regulates both motor and non-motor performance: A high-density EEG study in Parkinson's disease. Arch Ital Biol 148, 279-288.

[152] Jordan N, Sagar HJ, Cooper JA (1992) Cognitive components of reaction time in Parkinson's disease. J Neurol Neurosurg Psychiatry 55, 658-664.

[153] Cunnington R, Iansek R, Bradshaw JL (1999) Movementrelated potentials in Parkinson's disease: External cues and attentional strategies. Mov Disord 14, 63-68.

[154] Dick JPR, Cowan JMA, Day BL, Berardelli A, Kachi T, Rothwell JC, Marsden CD (1984) The corticomotoneurone connection is normal in Parkinson's disease. Nature 310, 407-409.

[155] Pascual-Leone A, Valls-Solé J, Brasil-Neto JP, Cohen LG, Hallett M (1994) Akinesia in Parkinson's disease.
I. Shortening of simple reaction time with focal, singlepulse transcranial magnetic stimulation. Neurology $\mathbf{4 4}$, 884-891.

[156] Libet B, Wright EW, Gleason CA (1983) Preparationor intention-to-act, in relation to pre-event potentials recorded at the vertex. Electroencephalogr Clin Neurophysiol 56, 367-372.

[157] Jahanshahi M, Jenkins IH, Brown RG, Marsden CD, Passingham RE, Brooks DJ (1995) Self-initiated versus externally triggered movements: I. An investigation using measurement of regional cerebral blood flow with PET and movement-related potentials in normal and Parkinson's disease subjects. Brain 118, 913-933.

[158] Cunnington R, Iansek R, Bradshaw JL, Phillips JG (1995) Movement-related potentials in Parkinson's disease. Presence and predictability of temporal and spatial cues. Brain 118 (Pt 4), 935-950.

[159] Touge T, Werhahn KJ, Rothwell JC, Marsden CD (1995) Movement-related cortical potentials preceding repetitive and random-choice hand movements in Parkinson's disease. Ann Neurol 37, 791-799.

[160] Praamstra P, Meyer AS, Cools AR, Horstink MWIM, Stegeman DF (1996) Movement preparation in Parkinson's disease Time course and distribution of movementrelated potentials in a movement precueing task. Brain 119, 1689-1704.

[161] Filipović SR, Čovičković-Šternić N, Radović VM, Dragašević N, Stojanović-Svetel M, Kostić VS (1997) Correlation between Bereitschaftspotential and reaction time measurements in patients with Parkinson's disease measuring the impaired supplementary motor area function? J Neurol Sci 147, 177-183.

[162] Goldberg G (1985) Supplementary motor area structure and function: Review and hypotheses. Behav Brain Sci $\mathbf{8}$, 567-588.

[163] Cunnington R, Windischberger C, Deecke L, Moser E (2002) The preparation and execution of self-initiated and externally-triggered movement: A study of event-related fMRI. Neuroimage 15, 373-385.

[164] Wu T, Wang L, Hallett M, Chen Y, Li K, Chan P (2011) Effective connectivity of brain networks during self-initiated movement in Parkinson's disease. Neuroimage 55, 204-215.

[165] Sabatini U, Boulanouar K, Fabre N, Martin F, Carel C, Colonnese C, Bozzao L, Berry I, Montastruc JL, Chollet F, Rascol O (2000) Cortical motor reorganization in akinetic patients with Parkinson's disease: A functional MRI study. Brain 123 (Pt 2), 394-403.

[166] Yu H, Sternad D, Corcos DM, Vaillancourt DE (2007) Role of hyperactive cerebellum and motor cortex in Parkinson's disease. Neuroimage 35, 222-233.

[167] Wu T, Wang L, Hallett M, Li K, Chan P (2010) Neural correlates of bimanual anti-phase and in-phase movements in Parkinson's disease. Brain 133, 2394-2409.

[168] Defebvre L, Bourriez JL, Destée A, Guieu JD (1996) Movement related desynchronisation pattern preceding voluntary movement in untreated Parkinson's disease. $J$ Neurol Neurosurg Psychiatry 60, 307-312.

[169] Magnani G, Cursi M, Leocani L, Volonté MA, Locatelli T, Elia A, Comi G (1998) Event-related desynchronization to contingent negative variation and self-paced movement paradigms in Parkinson's disease. Mov Disord 13, 653-660.

[170] Kühn AA, Williams D, Kupsch A, Limousin P, Hariz M, Schneider G, Yarrow K, Brown P (2004) Event-related 
beta desynchronization in human subthalamic nucleus correlates with motor performance. Brain 127, 735-746.

[171] Ray NJ, Jenkinson N, Wang S, Holland P, Brittain JS, Joint C, Stein JF, Aziz T (2008) Local field potential beta activity in the subthalamic nucleus of patients with Parkinson's disease is associated with improvements in bradykinesia after dopamine and deep brain stimulation. Exp Neurol 213, 108-113.

[172] Tinkhauser G, Pogosyan A, Tan H, Herz DM, Kühn AA, Brown P (2017) Beta burst dynamics in Parkinson's disease OFF and ON dopaminergic medication. Brain 140, 2968-2981.

[173] Torrecillos F, Tinkhauser G, Fischer P, Green AL, Aziz TZ, Foltynie T, Limousin P, Zrinzo L, Ashkan K, Brown P, Tan H (2018) Modulation of beta bursts in the subthalamic nucleus predicts motor performance. J Neurosci 38, 8905-8917.

[174] Tinkhauser G, Torrecillos F, Pogosyan A, Mostofi A, Bange M, Fischer P, Tan H, Hasegawa H, Glaser M, Muthuraman M, Groppa S, Ashkan K, Pereira EA, Brown $P$ (2020) The cumulative effect of transient synchrony states on motor performance in Parkinson's disease. $J$ Neurosci 40, 1571-1580.

[175] Brown P, Marsden CD (1998) What do the basal ganglia do? Lancet 351, 1801-1804.

[176] Wang H, Lees A, Brown P (1999) Impairment of EEG desynchronisation before and during movement and its relation to bradykinesia in Parkinson's disease. J Neurol Neurosurg Psychiatry 66, 442-446.

[177] Brown P, Oliviero A, Mazzone P, Insola A, Tonali P, Di Lazzaro V (2001) Dopamine dependency of oscillations between subthalamic nucleus and pallidum in Parkinson's disease. J Neurosci 21, 1033-1038.

[178] Brown P, Marsden CD (1999) Bradykinesia and impairment of EEG desynchronization in Parkinson's disease. Mov Disord 14, 423-429.

[179] George JS, Strunk J, Mak-McCully R, Houser M, Poizner H, Aron AR (2013) Dopaminergic therapy in Parkinson's disease decreases cortical beta band coherence in the resting state and increases cortical beta band power during executive control. Neuroimage Clin 3, 261-270.

[180] Canessa A, Pozzi NG, Arnulfo G, Brumberg J, Reich MM, Pezzoli G, Ghilardi MF, Matthies C, Steigerwald F, Volkmann J, Isaias IU (2016) Striatal dopaminergic innervation regulates subthalamic beta-oscillations and cortical-subcortical coupling during movements: Preliminary evidence in subjects with Parkinson's disease. Front Hum Neurosci 10, 611.

[181] Williams D, Kühn A, Kupsch A, Tijssen M, van Bruggen G, Speelman H, Hotton G, Yarrow K, Brown P (2003) Behavioural cues are associated with modulations of synchronous oscillations in the human subthalamic nucleus. Brain 126, 1975-1985.

[182] Mirabella G, Iaconelli S, Modugno N, Giannini G, Lena F, Cantore G (2013) Stimulation of subthalamic nuclei restores a near normal planning strategy in Parkinson's patients. PLoS One 8, e62793.

[183] Singh A, Levin J, Mehrkens JH, Bötzel K (2011) Alpha frequency modulation in the human basal ganglia is dependent on motor task. Eur J Neurosci 33, 960-967.

[184] Pani P, Di Bello F, Brunamonti E, D'Andrea V, Papazachariadis O, Ferraina S (2014) Alpha- and betaband oscillations subserve different processes in reactive control of limb movements. Front Behav Neurosci 8, 383.
[185] Alegre M, Valencia M (2013) Oscillatory activity in the human basal ganglia: More than just beta, more than just Parkinson's disease. Exp Neurol 248, 183-186.

[186] Joundi RA, Brittain J-S, Green AL, Aziz TZ, Brown P, Jenkinson N (2012) Oscillatory activity in the subthalamic nucleus during arm reaching in Parkinson's disease. Exp Neurol 236, 319-326.

[187] Alberts JL, Saling M, Adler CH, Stelmach GE (2000) Disruptions in the reach-to-grasp actions of Parkinson's patients. Exp Brain Res 134, 353-362.

[188] Schettino LF, Rajaraman V, Jack D, Adamovich SV, Sage J, Poizner H (2004) Deficits in the evolution of hand preshaping in Parkinson's disease. Neuropsychologia 42, 82-94.

[189] Snider J, Lee D, Harrington DL, Poizner H (2014) Scaling and coordination deficits during dynamic object manipulation in Parkinson's disease. J Neurophysiol 112, 300-315.

[190] Negrotti A, Secchi C, Gentilucci M (2005) Effects of disease progression and L-dopa therapy on the control of reaching-grasping in Parkinson's disease. Neuropsychologia 43, 450-459.

[191] Weiss PH, Dafotakis M, Metten L, Noth J (2009) Distal and proximal prehension is differentially affected by Parkinson's disease. J Neurol 256, 450.

[192] Rizzolatti G, Luppino G, Matelli M (1998) The organization of the cortical motor system: New concepts. Electroencephalogr Clin Neurophysiol 106, 283-296.

[193] Vissani M, Palmisano C, Volkmann J, Pezzoli G, Micera S, Isaias IU, Mazzoni A (2021) Impaired reach-to-grasp kinematics in parkinsonian patients relates to dopaminedependent, subthalamic beta bursts. NPJ Parkinsons Dis 7, 1-10.

[194] Castiello U, Bennett K, Bonfiglioli C, Lim S, Peppard RF (1999) The reach-to-grasp movement in Parkinson's disease: Response to a simultaneous perturbation of object position and object size. Exp Brain Res 125, 453-462.

[195] Rand MK, Smiley-Oyen AL, Shimansky YP, Bloedel JR, Stelmach GE (2006) Control of aperture closure during reach-to-grasp movements in Parkinson's disease. Exp Brain Res 168, 131-142.

[196] Jeannerod M(1988) The neural and behavioural organization of goal-directed movements, Clarendon Press/Oxford University Press, New York, NY, US.

[197] Gordon J, Ghilardi MF, Ghez C (1994) Accuracy of planar reaching movements. Exp Brain Res 99, 97-111.

[198] Chieffi S, Gentilucci M (1993) Coordination between the transport and the grasp components during prehension movements. Exp Brain Res 94, 471-477.

[199] Ansuini C, Begliomini C, Ferrari T, Castiello U (2010) Testing the effects of end-goal during reach-to-grasp movements in Parkinson's disease. Brain Cogn 74, 169-177.

[200] Castiello U, Stelmach GE, Lieberman AN (1993) Temporal dissociation of the prehension pattern in Parkinson's disease. Neuropsychologia 31, 395-402.

[201] Bertram CP, Lemay M, Stelmach GE (2005) The effect of Parkinson's disease on the control of multi-segmental coordination. Brain Cogn 57, 16-20.

[202] Molina-Vilaplana J, Contreras-Vidal JL, HerreroEzquerro MT, Lopez-Coronado J (2009) A model for altered neural network dynamics related to prehension movements in Parkinson disease. Biol Cybern 100, 271-287. 
[203] Straulino E, Scaravilli T, Castiello U (2015) Social intentions in Parkinson's disease patients: A kinematic study. Cortex 70, 179-188.

[204] Straulino E, Scaravilli T, Castiello U (2016) Dopamine depletion affects communicative intentionality in Parkinson's disease patients: Evidence from action kinematics. Cortex 77, 84-94.

[205] Straulino E, Scaravilli T, Bulgheroni M, D'Amico E, Castiello U (2016) It's all in the type of the task: Dopamine modulates kinematic patterns during competitive vs. cooperative interaction in Parkinson's disease. Neuropsychologia 93, 106-115.

[206] Lefaucheur J-P, Drouot X, Von Raison F, MénardLefaucheur I, Cesaro P, Nguyen J-P (2004) Improvement of motor performance and modulation of cortical excitability by repetitive transcranial magnetic stimulation of the motor cortex in Parkinson's disease. Clin Neurophysiol 115, 2530-2541.

[207] Khedr EM, Rothwell JC, Shawky OA, Ahmed MA, Hamdy A (2006) Effect of daily repetitive transcranial magnetic stimulation on motor performance in Parkinson's disease. Mov Disord 21, 2201-2205.

[208] Grüner U, Eggers C, Ameli M, Sarfeld A-S, Fink GR, Nowak DA (2010) $1 \mathrm{~Hz}$ rTMS preconditioned by tDCS over the primary motor cortex in Parkinson's disease: Effects on bradykinesia of arm and hand. J Neural Transm 117, 207-216.

[209] Thanakamchokchai J, Tretriluxana J, Pakaprot N, Pisarnpong A, Fisher BE (2020) Effects of high-frequency repetitive transcranial magnetic stimulation on reach-tograsp performance in individuals with Parkinson's disease: A preliminary study. Exp Brain Res 238, 1827-1837.

[210] Palomar FJ, Conde V, Carrillo F, Fernéndez-del-Olmo M, Koch G, Mir P (2013) Parieto-motor functional connectivity is impaired in Parkinson's disease. Brain Stimul 6, 147-154.

[211] Beretta VS, Conceição NR, Nóbrega-Sousa P, OrcioliSilva D, Dantas LKBF, Gobbi LTB, Vitório R (2020) Transcranial direct current stimulation combined with physical or cognitive training in people with Parkinson's disease: A Systematic review. J Neuroeng Rehabil 17, 74.

[212] Simpson MW, Mak M (2020) The effect of transcranial direct current stimulation on upper limb motor performance in Parkinson's disease: A systematic review. $J$ Neurol 267, 3479-3488.

[213] Kami AT, Sadler C, Nantel J, Carlsen AN (2018) Transcranial direct current stimulation (TDCS) over supplementary motor area (SMA) improves upper limb movement in individuals with Parkinson's disease. J Exercise Mov Sport (SCAPPS refereed abstracts repository) 50, 36-36.

[214] Tamés G, Kelemen A, Radics P, Valélik I, Heldman D, Klivényi P, Vécsei L, Hidasi E, Halész L, Kis D, Barsi P, Golopencza P, Erőss L (2016) Effect of subthalamic stimulation on distal and proximal upper limb movements in Parkinson's disease. Brain Res 1648, 438-444.

[215] Israeli-Korn SD, Hocherman S, Hassin-Baer S, Cohen OS, Inzelberg R (2013) Subthalamic nucleus deep brain stimulation does not improve visuo-motor impairment in parkinson's disease. PLoS One 8, e65270.

[216] Pötter-Nerger M, Habben A, Herzog J, Falk D, Mehdorn MH, Deuschl G, Volkmann J (2013) Kinematic effects of subthalamic stimulation on reach-to-grasp movements in Parkinson's disease. Parkinsonism Relat Disord 19, 32-36.

[217] Dafotakis M, Fink GR, Allert N, Nowak DA (2008) The impact of subthalamic deep brain stimulation on bradykinesia of proximal and distal upper limb muscles in Parkinson's disease. J Neurol 255, 429-437.

[218] Agostino R, Dinapoli L, Modugno N, Iezzi E, Romanelli P, Berardelli A (2008) Effects of unilateral subthalamic deep brain stimulation on contralateral arm sequential movements in Parkinson's disease. J Neurol Neurosurg Psychiatry 79, 76-78.

[219] David FJ, Goelz LC, Tangonan RZ, Metman LV, Corcos DM (2018) Bilateral deep brain stimulation of the subthalamic nucleus increases pointing error during memory-guided sequential reaching. Exp Brain Res 236, 1053-1065.

[220] Neumann W-J, Schroll H, de Almeida Marcelino AL, Horn A, Ewert S, Irmen F, Krause P, Schneider G-H, Hamker F, Kühn AA (2018) Functional segregation of basal ganglia pathways in Parkinson's disease. Brain 141, 2655-2669.

[221] Spraker MB, Yu H, Corcos DM, Vaillancourt DE (2007) Role of individual basal ganglia nuclei in force amplitude generation. J Neurophysiol 98, 821-834.

[222] Tunik E, Feldman AG, Poizner H (2007) Dopamine replacement therapy does not restore the ability of Parkinsonian patients to make rapid adjustments in motor strategies according to changing sensorimotor contexts. Parkinsonism Relat Disord 13, 425-433.

[223] Nowak DA, Tisch S, Hariz M, Limousin P, Topka H, Rothwell JC (2006) Sensory timing cues improve akinesia of grasping movements in Parkinson's disease: A comparison to the effects of subthalamic nucleus stimulation. Mov Disord 21, 166-172.

[224] Colnat-Coulbois S, Gauchard GC, Maillard L, Barroche G, Vespignani H, Auque J, Perrin PP (2005) Bilateral subthalamic nucleus stimulation improves balance control in Parkinson's disease. J Neurol Neurosurg Psychiatry 76, 780-787.

[225] Schettino LF, Van Erp E, Hening W, Lessig S, Song D, Barba D, Poizner H (2009) Deep brain stimulation of the subthalamic nucleus facilitates coordination of hand preshaping in Parkinson's disease. Int J Neurosci 119, 1905-1924.

[226] Grafton ST, Turner RS, Desmurget M, Bakay R, Delong M, Vitek J, Crutcher M (2006) Normalizing motor-related brain activity: Subthalamic nucleus stimulation in Parkinson disease. Neurology 66, 1192-1199.

[227] Maschke M, Tuite PJ, Pickett K, Wächter T, Konczak J (2005) The effect of subthalamic nucleus stimulation on kinaesthesia in Parkinson's disease. J Neurol Neurosurg Psychiatry 76, 569-571.

[228] Jasielski PP, Piędel F, Szumna K, Madras D, Rocka A (2020) Deep brain stimulation in Parkinson's disease - the review. J Educ Health Sport 10, 41-46.

[229] Jakobs M, Fomenko A, Lozano AM, Kiening KL (2019) Cellular, molecular, and clinical mechanisms of action of deep brain stimulation-a systematic review on established indications and outlook on future developments. EMBO Mol Med 11, e9575.

[230] Vissani M, Isaias IU, Mazzoni A (2020) Deep brain stimulation: A review of the open neural engineering challenges. J Neural Eng 17, 051002.

[231] Moroney R, Heida C, Geelen J (2008) Increased bradykinesia in Parkinson's disease with increased movement complexity: Elbow flexion-extension movements. J Comput Neurosci 25, 501.

[232] Rubin JE, Terman D (2004) High frequency stimulation of the subthalamic nucleus eliminates pathological thalamic 
rhythmicity in a computational model. J Comput Neurosci 16, 211-235.

[233] Benabid AL, Benazzous A, Pollak P (2002) Mechanisms of deep brain stimulation. Mov Disord 17(Suppl 3), S73-74.

[234] Chakravarthy VS, Joseph D, Bapi RS (2010) What do the basal ganglia do? A modeling perspective. Biol Cybern 103, 237-253.

[235] Redgrave P, Prescott TJ, Gurney K (1999) The basal ganglia: A vertebrate solution to the selection problem? Neuroscience 89, 1009-1023.

[236] Prescott TJ, Montes Gonzélez FM, Gurney K, Humphries MD, Redgrave P (2006) A robot model of the basal ganglia: Behavior and intrinsic processing. Neural Netw 19, 31-61.

[237] Yttri EA, Dudman JT (2016) Opponent and bidirectional control of movement velocity in the basal ganglia. Nature 533, 402-406.

[238] Nicola SM (2010) The flexible approach hypothesis: Unification of effort and cue-responding hypotheses for the role of nucleus accumbens dopamine in the activation of reward-seeking behavior. $J$ Neurosci 30, 16585-16600.

[239] Cutsuridis V, Perantonis S (2006) A neural network model of Parkinson's disease bradykinesia. Neural Netw 19, 354-374.

[240] Cutsuridis V (2011) Origins of a repetitive and cocontractive biphasic pattern of muscle activation in Parkinson's disease. Neural Netw 24, 592-601.

[241] Contreras-Vidal JL, Grossberg S, Bullock D (1997) A neural model of cerebellar learning for arm movement control: Cortico-spino-cerebellar dynamics. Learn Mem 3, 475-502.

[242] Marsden CD (1989) Slowness of movement in Parkinson's disease. Mov Disord 4, S26-S37.

[243] Falotico E, Vannucci L, Ambrosano A, Albanese U, Ulbrich S, Vasquez Tieck JC, Hinkel G, Kaiser J, Peric I, Denninger O, Cauli N, Kirtay M, Roennau A, Klinker G, Von Arnim A, Guyot L, Peppicelli D, Martínez-Cañada P, Ros E, Maier P, Weber S, Huber M, Plecher D, Röhrbein F, Deser S, Roitberg A, van der Smagt P, Dillman R, Levi P, Laschi C, Knoll AC, Gewaltig M-O (2017) Connecting artificial brains to robots in a comprehensive simulation framework: The neurorobotics platform. Front Neurorobot 11, 2.

[244] Falotico E, Kalidindi H-T (2020) Neurorobotics: Motor control principles for robots. In AI-Enabled health care: From decision support to autonomous robots. (Gruppo Nazionale di Bioingegneria; 39), Patron, Bologna, pp. 243-263.

[245] Rubin JE (2017) Computational models of basal ganglia dysfunction: The dynamics is in the details. Curr Opin Neurobiol 46, 127-135.

[246] Suryanarayana SM, Hellgren Kotaleski J, Grillner S, Gurney KN (2019) Roles for globus pallidus externa revealed in a computational model of action selection in the basal ganglia. Neural Networks 109, 113-136.

[247] Nambu A (2004) A new dynamic model of the corticobasal ganglia loop. Prog Brain Res 143, 461-466. 